Original Research Paper

\title{
The Complex Probability Paradigm and Analytic Linear Prognostic for Vehicle Suspension Systems
}

\author{
Abdo Abou Jaoude \\ Department of Mathematics and Statistics, Faculty of Natural and Applied Sciences, Notre Dame University-Louaize, Lebanon
}

\author{
Article history \\ Received: 20-02-2015 \\ Revised: 05-04-2015 \\ Accepted: 06-04-2015 \\ Email: abdoaj@idm.net.lb
}

\begin{abstract}
The Andrey N. Kolmogorov's system of axioms can be extended to encompass the imaginary set of numbers and this by adding to his original five axioms an additional three axioms. Hence, any experiment can thus be executed in what is now the complex set $C$ which is the sum of the real set $R$ with its corresponding real probability and the imaginary set $M$ with its corresponding imaginary probability. The objective here is to evaluate the complex probabilities by considering supplementary new imaginary dimensions to the event occurring in the "real" laboratory. Whatever the probability distribution of the input random variable in $R$ is, the corresponding probability in the whole set $C$ is always one, so the outcome of the random experiment in $C$ can be predicted totally. The result indicates that chance and luck in $R$ is replaced now by total determinism in $C$. This is the consequence of the fact that the probability in $C$ is got by subtracting the chaotic factor from the degree of our knowledge of the system. This novel complex probability paradigm will be applied to the concepts of degradation and the remaining useful lifetime of a vehicle suspension system, thus to the field of prognostic.
\end{abstract}

Keywords: Extended Kolmogorov's Axioms, Complex Set, Probability Norm, Degree of Our Knowledge, Chaotic Factor, Linear Damage, Degradation, Remaining Useful Lifetime, Prognostic

\section{Introduction}

"An intellect which at any given moment knew all the forces that animate Nature and the mutual positions of the beings that comprise it, if this intellect were vast enough to submit its data to analysis, could condense into a single formula the movement of the greatest bodies of the universe and that of the lightest atom: For such intellect nothing could be uncertain; and the future just like the past would be present before its eyes."

Marquis Pierre-Simon de Laplace

"The Divine Spirit found a sublime outlet in that wonder of analysis, that portent of the ideal world, that amphibian between being and not-being, which we call the imaginary root of negative unity."

Gottfried Wilhelm Von Leibniz

Recent developments in system design technology like in aerospace, defense, petro-chemistry and automobiles, are represented earlier in literature by simulated models during the conception step and this to ensure the high availability of the industrial systems. Knowing that, the integration of diagnostic-prognostic models in these industrial systems is facilitated by these developments. In fact, the monitoring of the degradation indicators is used indirectly in failure prognostic and is just a measurement of an unwanted situation. Therefore, the diagnostic is not only a failure detection procedure but it also indicates the actual state and the historic of the system. Hence, a predictive maintenance is done by the subsequent prognostic model. Consequently, from a predefined threshold of degradation, the Remaining Useful Lifetime (RUL) is estimated. Based on a physical dynamic vehicle suspension system, this research paper elaborates a procedure to create a failure prognostic model (Abou Jaoude et al., 2010a).

Moreover, predicting the remaining useful lifetime of industrial systems becomes currently an important aim for industrialists knowing that the failure which can occur suddenly is generally very expensive at the level of reparation, of production interruption and is bad for 
reputation. The classical strategies of maintenance (Vachtsevanos et al., 2006) are no more efficient and practical because they do not take into consideration the instantaneous evolving product state, so it is important to understand the product in real time in order to prevent a failure during operation. In fact, we introduce a prognostic approach that seeks to provide an intelligent maintenance.

A proposed analytic prognostic methodology based on some laws of damage in fracture mechanics is developed here. The damages are generally: Crack propagation, corrosions, chloride attack, creep, excessive deformation and deflection and damage accumulation. Whenever their analytic laws are available, the advantage of a prognostic approach based on a known damage law for a mechanical system is that it is adaptable to new situations and useful in determining the $R U L$ of the system (Abou Jaoude and El-Tawil, 2013a; El-Tawil and Abou Jaoude, 2013).

The procedure proposed in this study belongs to the model based prognosis approach related to the physical model. It is focused on developing and implementing effective diagnostic and prognostic technologies with the ability to detect faults in the early stages of degradation. Early detection and analysis may lead to better prediction and end of life estimations by tracking and modeling the degradation process. The idea is to use these estimations to make accurate and precise prediction of the time to failure of components. Early detection also helps avoid catastrophic failures (Abou Jaoude and El-Tawil, 2013b).

Any prognostic methodology must lie on a type of damage. In this research paper, the case of fatigue degradation has been chosen due to the fact that it can be mathematically formulated by available analytic laws of degradation such as Paris-Erdogan's law and PalmgrenMiner's law for cumulative damage (Abou Jaoude, 2012; Abou Jaoude, 2013a).

In automobile industry, like for example the suspension component, this approach shows its importance for the same earlier reasons as it was explained in the paper (Abou Jaoude, 2015). In vehicle suspension system study the results of model simulations are done for three cases of road profile excitations.

Furthermore, all our work in classical probability theory is to compute probabilities. The original idea in this study is to add new dimensions to our random experiment and this will make the work deterministic. In fact, the probability theory is a nondeterministic theory by nature; that means that the outcome of the events is due to chance and luck. By adding new dimensions to the event, we make the work deterministic and hence a random experiment will have a certain outcome in the complex set of probabilities C. It is of great importance that the stochastic system becomes totally predictable since we will be totally knowledgeable to predict the outcome of chaotic and random events that occur in nature like for example in statistical mechanics or in all stochastic processes. Therefore the work that should be done is to add to the real set of probabilities $\mathrm{R}$, the contributions of $\mathrm{M}$ which is the imaginary set of probabilities, that makes the event in $\mathrm{C}=\mathrm{R}+\mathrm{M}$ deterministic. If this is found to be fruitful, then a new theory in statistical sciences and prognostic is elaborated and this to understand deterministically those phenomena that used to be random phenomena in R. This is what I called "the complex probability paradigm" that was initiated and elaborated in four previous papers (Abou Jaoude et al., 2010b; Abou Jaoude, 2013b; 2013c; 2014).

Moreover, although the analytic laws are deterministic and very well-known (Abou Jaoude, 2015) but there are random factors (temperature, humidity, applied load location, water action, etc...) that affect the system and make its degradation function deviate from its calculated trajectory predefined by these laws. An updated follow-up of the degradation behavior with time or cycle number is done by the system failure probability. An accentuated effect of chaos on the system leads to a bigger jump in the degradation trajectory and hence to a greater probability of failure.

Consequently, my purpose in this current work is to link the complex probability paradigm to the vehicle suspension system analytic and linear prognostic. By calculating the parameters of the new model, we will be able to determine the magnitude of the chaotic factor, the degree of our knowledge, the complex probability and the failure probability of the suspension system, which are all functions of the system degradation subject to chaos and random effects.

Finally, this study is organized as follows: Firstly the analytic prognostic model of fatigue for vehicle suspension systems is recapitulated in the linear cumulative damage case, secondly the extended Kolmogorov's axioms with their original parameters and interpretation are presented, thirdly the complex probability paradigm applied to prognostic is elaborated, fourthly the simulations of the new model for the three roads modes are illustrated and finally a comprehensive conclusion and perspectives end this research.

\section{The Analytic and Linear Prognostic Model for Vehicle Suspension Systems}

The purpose of my previous paper (Abou Jaoude, 2015) was to create a model of prognostic capable of predicting the degradation trajectories of a vehicle suspension system under a given environment and starting from an initial known damage.

The fatigue failure is one of the famous damage phenomena in mechanical systems like in vehicles where the suspension systems are subject to the fluctuation of roads stresses and loads $\sigma_{j}$ (Lemaitre and Chaboche, 1990). This type of loadings leads to crack propagation that can accelerate rapidly. Usually, micro-cracks exist originally in the materials due to fabrication process where stresses 
remain after manufacture. These micro-cracks are detected and measured and denoted by $a_{0}$. The advantage of the choice of fatigue damage for the developed prognostic methodology was that it is a failure mechanism very well studied in literature and described under many known analytic laws. This mechanism has relatively the simplest formulation in comparison to the other damage phenomena. The fatigue characterizes the main failure cause of industrial equipment (Abou Jaoude et al., 2011).

An analytic linear prognostic model was introduced in the previous research paper (Abou Jaoude, 2015) that permits to predict the Remaining Useful Lifetime (RUL) of a dynamic suspension system. This model considered the fatigue as a damage parameter and hence it was based on well-known laws of damage like Paris-Erdogan and Palmgren-Miner laws. An index of degradation was derived that varies from zero to one. My proposed model was based on the link between this index $D$ and the crack length $a$. Failure is produced when $a$ reaches a critical length $a_{C}$ after $N_{\mathrm{C}}$ critical load cycles. Hence, my model was given by a simple function relating the instantaneous degradation to actual crack length $a_{N}$ as a measurement of actual damage after $N$ load cycles. It was assumed that $a_{C}=e / 8$, where $e$ is the width of the mechanical component of the suspension.

The aim was to evaluate the evolution of the system lifetime at each instant. For this purpose the degradation trajectories had been used in terms of cycle numbers or the time of operation. From these degradation trajectories, the RULs variations were deduced. The prognostic of a complex system can be deduced from the prognostic of its sub-systems when their damage laws are available. To demonstrate the effectiveness of the model, an industrial example had been considered in the simulation in this previous paper (Abou Jaoude, 2015). This example was the vehicle suspension system where three modes of road profiles (severe, fair and good conditions) were simulated and examined. In such industrial system, this model proved that it is very convenient and it provides a useful tool for a prognostic analysis. Moreover, it is less expensive than other models that need a large number of data and measurements. Hence, the prognostic analytic linear model is presented by the general function given by Equation 1:

$$
\begin{aligned}
& D_{N}=D(N)=P_{r o g}\left(a_{N}\right)=\frac{a_{N-1}}{a_{C}-a_{0}} \\
& +\frac{C}{a_{C}-a_{0}} \cdot Y\left(a_{N-1}\right)^{m} \cdot\left(\sqrt{\pi \cdot a_{N-1}}\right)^{m} \cdot \sigma_{j}^{m}
\end{aligned}
$$

And therefore, the degradation trajectories $D(N)$ as well as $R U L(N)$ along the total number of loading cycles $N$ can be drawn.

\section{Flowchart of the Analytic Linear Prognostic Model}

The following flowchart summarizes all the procedures of the proposed model Fig. 1 (Abou Jaoude, 2015):

\section{Results of the Model Simulations}

Note that in the current paper some of the initial parameters used: $a_{0}$ (initial micro-crack), $e$ (width of the suspension mechanical component), $C$ (environment effect parameter), are different from those adopted in the previous paper (Abou Jaoude, 2015), keeping always the three load profiles for the three roads modes (severe, fair and good) the same. Also, $m$ (the suspension material parameter) is always equal to 2 in both research works. Therefore, the simulations yield somewhat different curves shapes for degradation and $R U L$.

In mode 1 case (severe), it is noted that (Fig. 2) for $N$ $=6,836,000$ cycles, the degradation $D_{\mathrm{N}}$ reaches the critical value $D_{\mathrm{C}}=1$. The deduced lifetime of the suspension is $6,836,000$ cycles of road excitation in mode 1 . Moreover, the first sign of damage appears at about 2,000,000 cycles. Starting from 6,000,000 cycles, the slope of the degradation curve becomes very acute; hence damage is increasing very fast.

In mode 2 case (fair), it is noted that (Fig. 3) for $N=$ $10,850,000$ cycles, the degradation $D_{\mathrm{N}}$ reaches the critical value $D_{\mathrm{C}}=1$. The deduced lifetime of the suspension is $10,850,000$ cycles of road excitation in mode 2. Moreover, the first sign of damage appears at about 2,500,000 cycles. Starting from 10,000,000 cycles, the slope of the degradation curve becomes very steep; hence damage is increasing very rapidly.

In mode 3 case (good), it is noted that (Fig. 4) for $N=$ $17,222,000$ cycles, the degradation $D_{\mathrm{N}}$ reaches the critical value $D_{\mathrm{C}}=1$. The deduced lifetime of the suspension is 17,222,000 cycles of road excitation in mode 3. Moreover, the first sign of damage appears at about 4,000,000 cycles. Starting from 15,000,000 cycles, the slope of the degradation curve becomes very acute; hence damage is increasing very quickly.

In addition, Fig. 5 recapitulates the three previous figures.

From the above, the three expected lifetimes are as follows: $N_{\mathrm{C} 1}=6,836,000$ cycles; $N_{\mathrm{C} 2}=10,850,000$ cycles; $N_{\mathrm{C} 3}=17,222,000$ cycles. Then, our prognostic procedure yields the Remaining Useful Lifetimes (RUL) for the three modes (Fig. 6) that can now be easily deduced from these three curves at any instant or any active cycle $N$ as follows:

$$
\begin{aligned}
& \text { For mode } 1: R U L_{1}(N)=N_{C 1}-N \\
& \text { For mode } 2: R U L_{2}(N)=N_{C 2}-N \\
& \text { For mode } 3: R U L_{3}(N)=N_{C 3}-N
\end{aligned}
$$

\section{The Original Andrey N. Kolmogorov's Set of Axioms}

The simplicity of Kolmogorov's system of axioms may be surprising. Let $E$ be a collection of elements 
$\left\{E_{1}, E_{2}, \ldots\right\}$ called elementary events and let $F$ be a set of subsets of $E$ called random events. The five axioms for a finite set $E$ are (Benton, 1966a; 1966b; Feller, 1968; Montgomery and Runger, 2003; Walpole et al., 2002):

- $F$ is a field of sets

- $F$ contains the set $E$
- A non-negative real number $P_{r o b}(A)$, called the probability of $A$, is assigned to each set $A$ in $F$. We have always $0 \leq P_{r o b}(A) \leq 1$

- $\quad P_{r o b}(E)$ equal 1

- If $A$ and $B$ have no elements in common, the number assigned to their union is:

$$
P_{r o b}(A \cup B)=P_{r o b}(A)+P_{r o b}(B)
$$

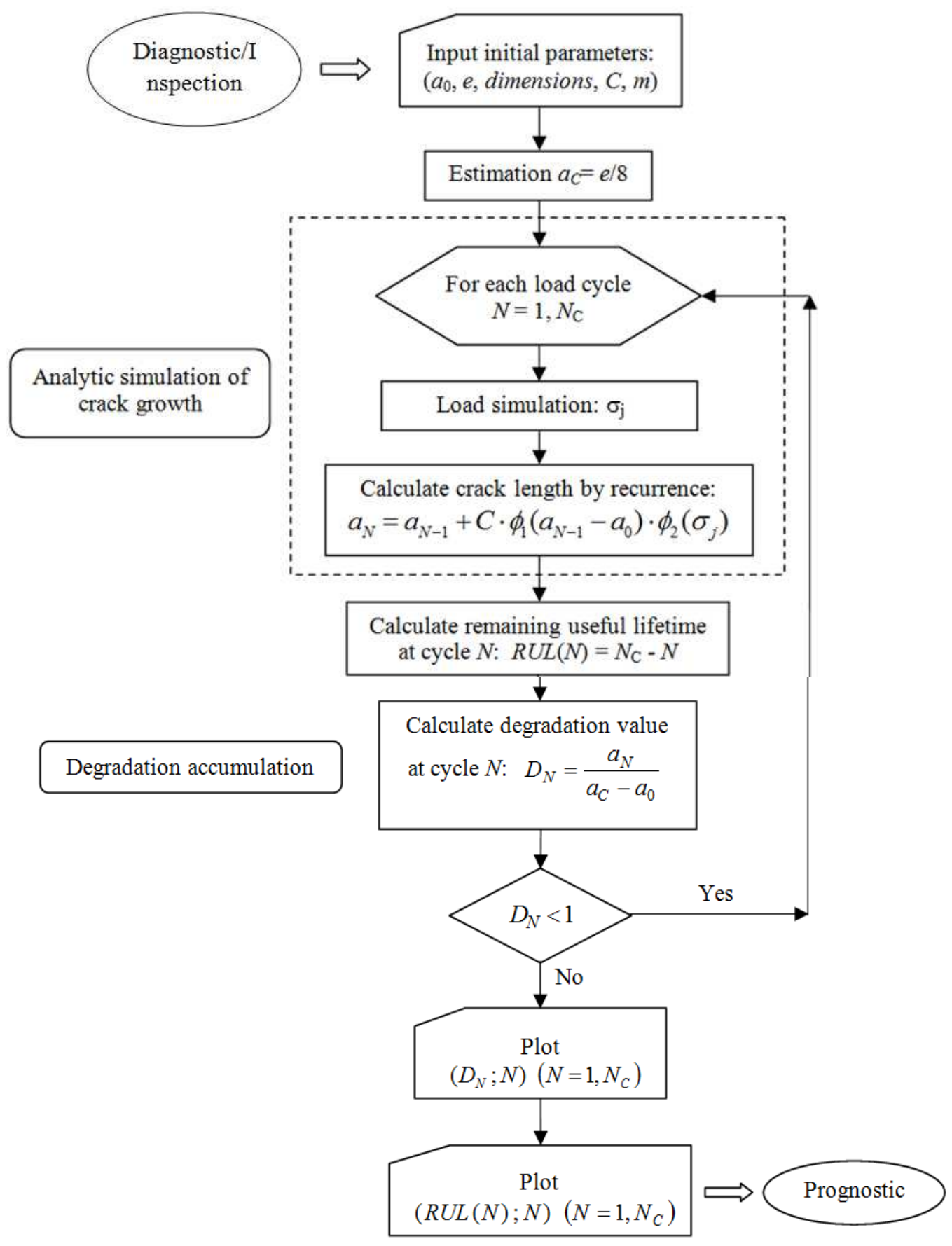

Fig. 1. Flowchart of the analytic linear prognostic model 


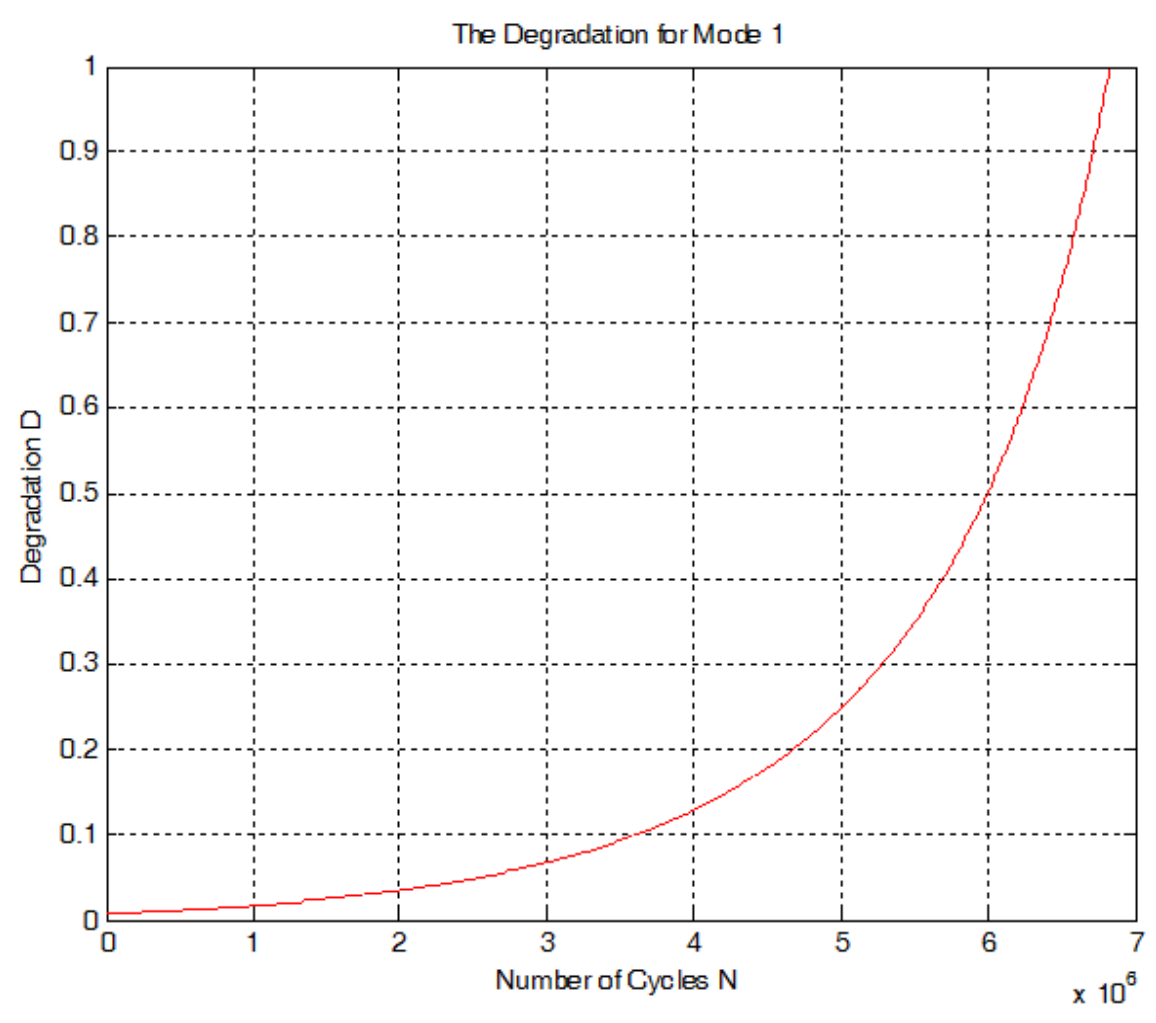

Fig. 2. Degradation trajectory for mode 1 (severe) of roads

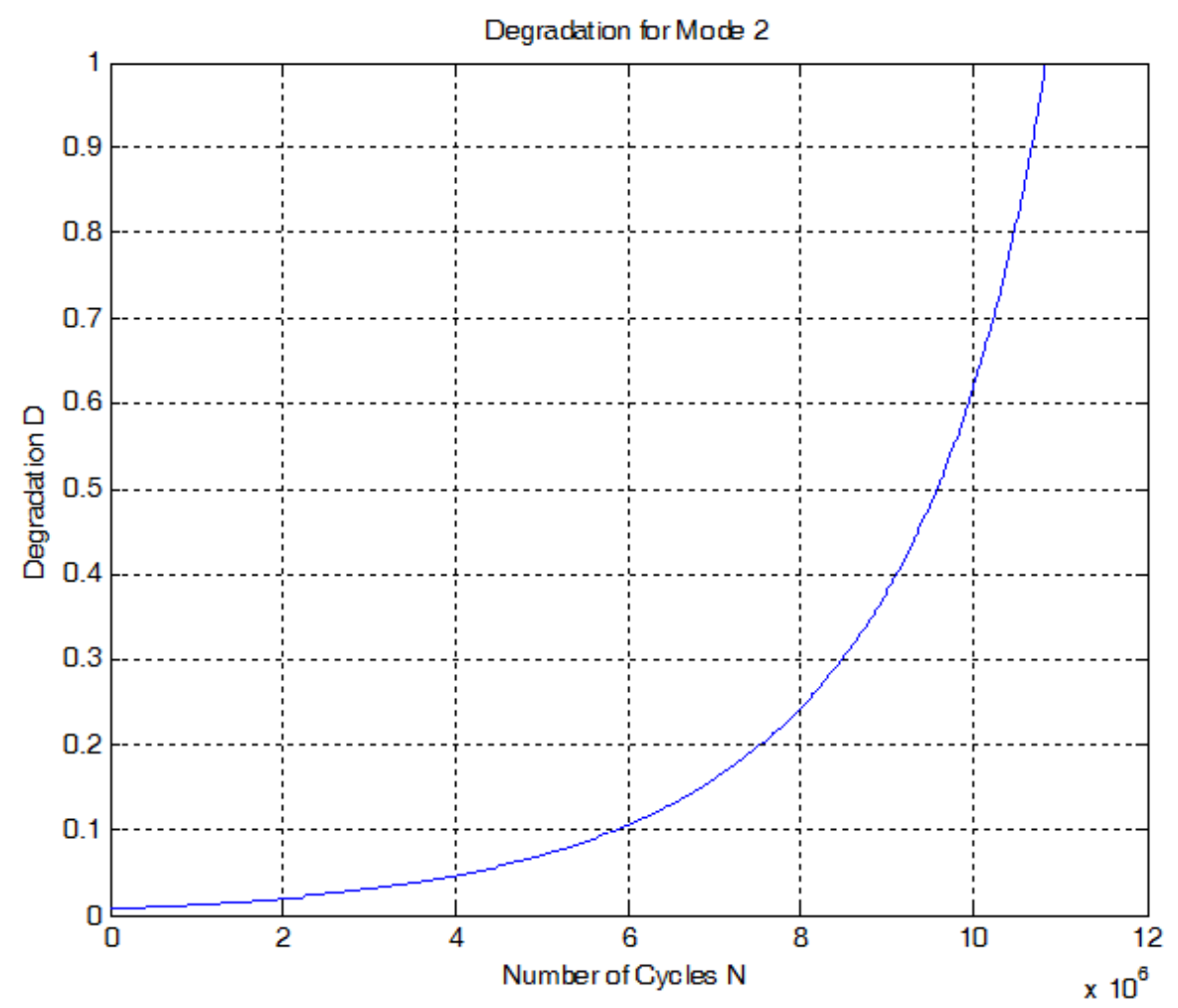

Fig. 3. Degradation trajectory for mode 2 (fair) of roads 


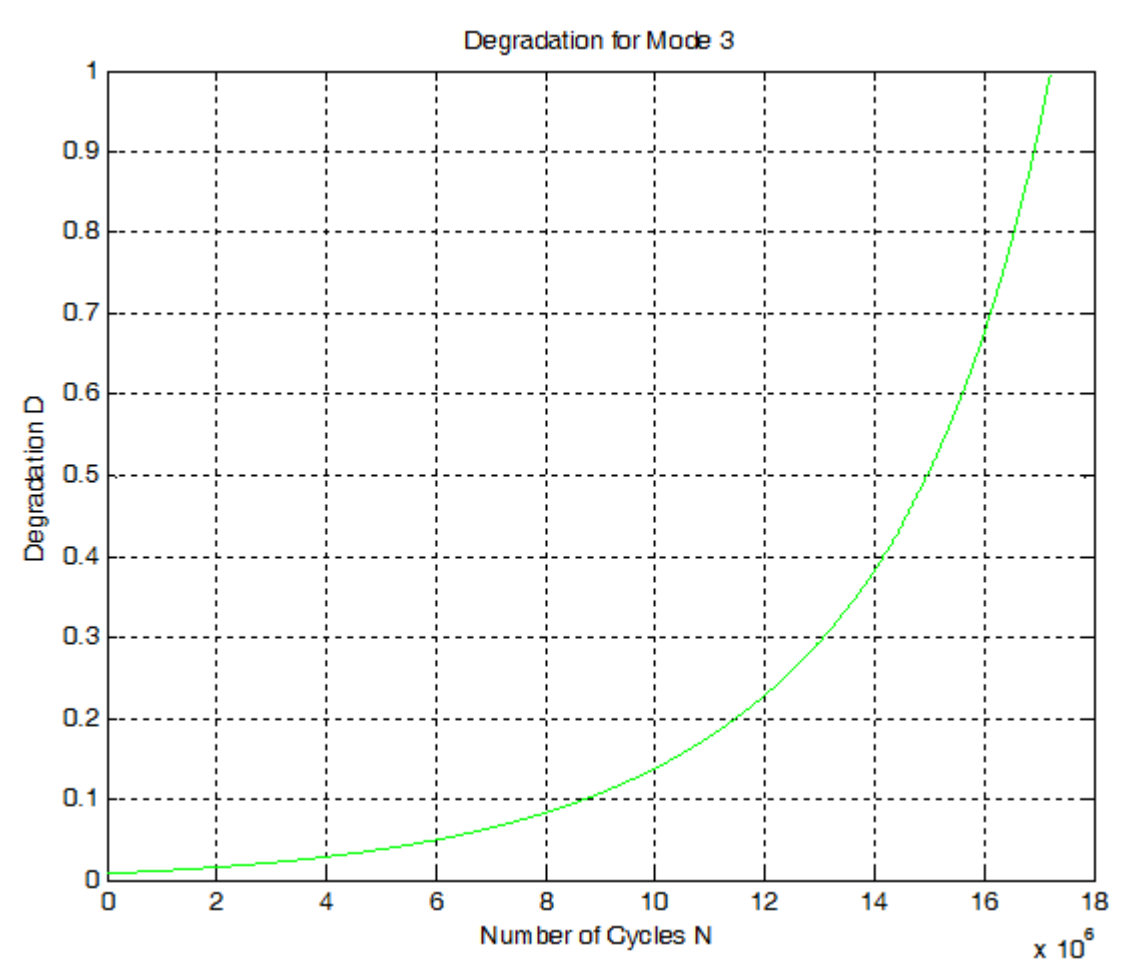

Fig. 4. Degradation trajectory for mode 3 (good) of roads

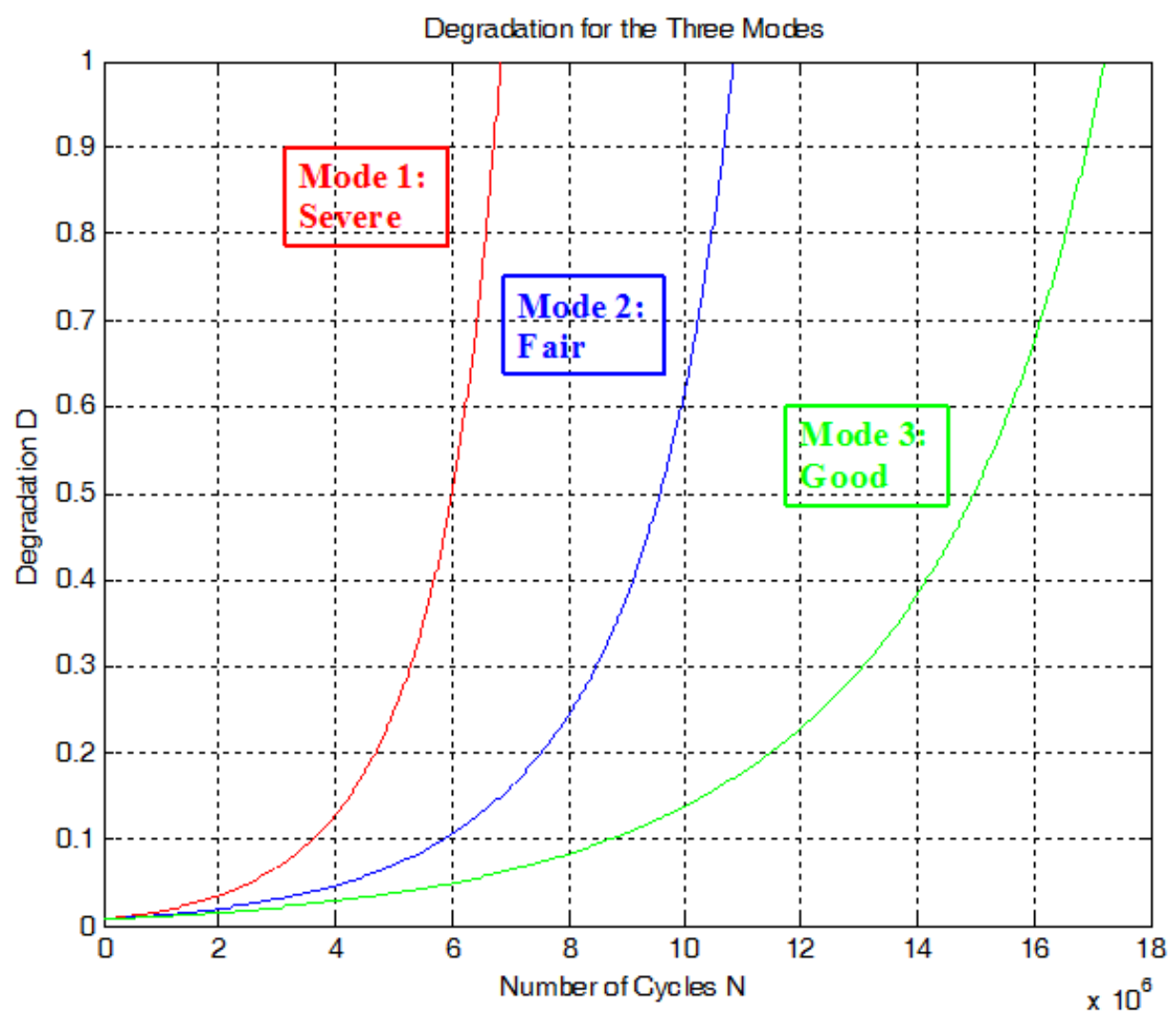

Fig. 5. Degradation trajectories for the three modes of roads 


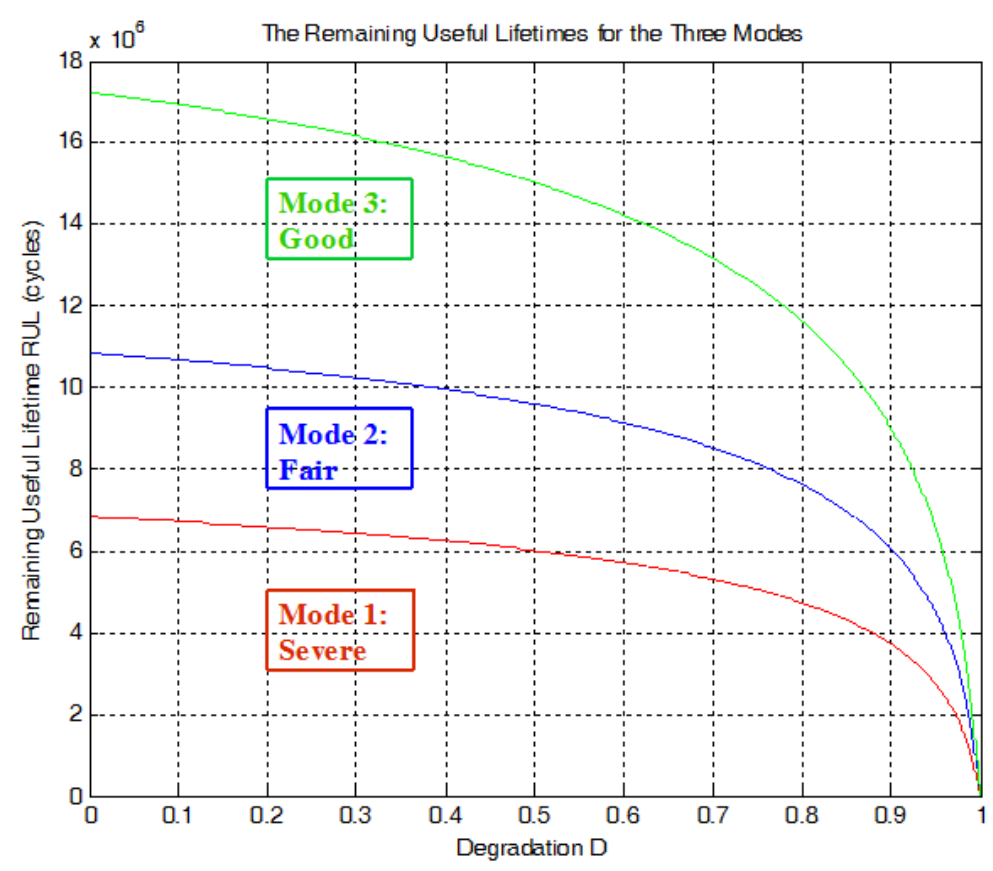

Fig. 6. RUL trajectories for the three modes of roads

Hence, we say that $A$ and $B$ are disjoint; otherwise, we have:

$$
P_{r o b}(A \cup B)=P_{r o b}(A)+P_{r o b}(B)-P_{r o b}(A \cap B)
$$

And we say also that $P_{r o b}(A \cap B)=P_{r o b}(A) \times P_{r o b}(B \mid A)$ $=P_{r o b}(B) \times P_{r o b}(A \mid B)$ which is the conditional probability. If both $A$ and $B$ are independent, then $P_{r o b}(A \cap B)=P_{r o b}(A) \times P_{r o b}(B)$.

\section{Adding the Imaginary Part $M$}

Now, we can add to this system of axioms an imaginary part such that:

- $\quad$ Let $P_{m}=i\left(1-P_{r}\right)$ be the probability of an associated event in $M$ (the imaginary part) to the event $A$ in $R$ (the real part). It follows that $P_{r}+P_{m} / i=1$ where $i^{2}=$ -1 (the imaginary number)

- We construct the complex number or vector $Z=P_{r}+$ $P_{m}=P_{r}+i\left(1-P_{r}\right)$ having a norm $|Z|$ such that:

$$
|Z|^{2}=P_{r}^{2}+\left(P_{m} / i\right)^{2}
$$

- Let $P$ c denote the probability of an event in the universe $C$ where $C=R+M$. We say that $P$ c is the probability of an event $A$ in $\mathrm{R}$ with its associated event in M such that:

$$
P c^{2}=\left(P_{r}+P_{m} / i\right)^{2}=|Z|^{2}-2 i P_{r} P_{m} \text { and is always equal to } 1
$$

We can see that the system of axioms defined by Kolmogorov could be hence expanded to take into consideration the set of imaginary probabilities by adding three new axioms (Abou Jaoude et al., 2010b; Abou Jaoude, 2013b; 2013c; 2014).

\section{The Purpose of Extending the Axioms}

It is apparent from the set of axioms that the addition of an imaginary part to the real event makes the probability of the event in $C$ always equal to 1 . In fact, if we begin to see the set of probabilities as divided into two parts, one real and the other imaginary, understanding will follow directly. The random event that occurs in $R$ (like tossing a coin and getting a head), has a correspondent probability $P_{r}$. Now, let $M$ be the set of imaginary probabilities and let $|Z|^{2}$ be the degree of our knowledge of this phenomenon. $P_{r}$ is always and according to Kolmogorov's axioms, the probability of an event.

A total ignorance of the set $M$ makes:

$$
P_{r}=0.5
$$

And $|Z|^{2}$ in this case is equal to: $1-2 P_{r}\left(1-P_{r}\right)=1-$ $(2 \times 0.5) \times(1-0.5)=0.5$.

Conversely, a total knowledge of the set in $R$ makes: $P_{r o b}($ event $)=1$ and $P_{m}=P_{r o b}($ imaginary part $)$ $=0$. Here we have $|Z|^{2}=1-(2 \times 1) \times(1-1)=1$ because the phenomenon is totally known, that is, its laws and 
variables are completely determined, hence; our degree of our knowledge of the system is 1 or $100 \%$.

Now, if we can tell for sure that an event will never occur i.e., like 'getting nothing' (the empty set), $P_{\mathrm{r}}$ is accordingly $=0$, that is the event will never occur in $R$. $P_{m}$ will be equal to $i\left(1-P_{r}\right)=i(1-0)=i$ and $|Z|^{2}=1-$ $(2 \times 0) \times(1-0)=1$, because we can tell that the event of getting nothing surely will never occur; thus, the Degree of Our Knowledge ( $D O K$ for short) of the system is 1 or 100\% (Abou Jaoude et al., 2010b).

We can infer that we have always:

$$
\begin{aligned}
& 0.5 \leq|Z|^{2} \leq 1 \text { since }|Z|^{2}=D O K=P_{r}^{2}+\left(P_{m} / i\right)^{2} \text { and } \\
& 0 \leq P_{r}, P_{m} / i \leq 1
\end{aligned}
$$

And what is important is that in all cases we have:

$$
\begin{aligned}
& P c^{2}=\left(P_{\mathrm{r}}+P_{m} / i\right)^{2}=|Z|^{2}-2 i P_{r} P_{m} \\
& =\left[P_{r}+\left(1-P_{r}\right)\right]^{2}=1^{2}=1
\end{aligned}
$$

In fact, according to an experimenter in $R$, the game is a game of luck: the experimenter doesn't know the output of the event. He will assign to each outcome a probability $P_{r}$ and he will say that the output is nondeterministic. But in the universe $C=R+M$, an observer will be able to predict the outcome of the game of chance since he takes into consideration the contribution of $M$, so we write:

$$
P \mathrm{c}^{2}=\left(P_{\mathrm{r}}+P_{\mathrm{m}} / i\right)^{2}
$$

Hence $P c$ is always equal to 1 . In fact, the addition of the imaginary set to our random experiment resulted to the abolition of ignorance and indeterminism. Consequently, the study of this class of phenomena in $C$ is of great usefulness since we will be able to predict with certainty the outcome of experiments conducted. In fact, the study in R leads to unpredictability and uncertainty. So instead of placing ourselves in $R$, we place ourselves in $C$ then study the phenomena, because in $C$ the contributions of $M$ are taken into consideration and therefore a deterministic study of the phenomena becomes possible. Conversely, by taking into consideration the contribution of the set $M$ we place ourselves in $C$ and by ignoring $M$ we restrict our study to nondeterministic phenomena in $R$ (Srinivasan and Mehata, 1988; Stewart, 2002; Van Kampen, 2006; Bell, 1992; Boursin, 1986; Dacunha-Castelle, 1996; Dalmedico-Dahan et al., 1992).

Moreover, it follows from the above definitions and axioms that (Abou Jaoude et al., 2010b):

$$
\begin{aligned}
2 i P_{r} P_{m}= & 2 i \times P_{r} \times i \times\left(1-P_{r}\right) \\
& =2 i^{2} \times P_{r} \times\left(1-P_{r}\right)=-2 P_{r}\left(1-P_{r}\right) \\
& =C h f
\end{aligned}
$$

$2 i P_{r} P_{m}$ will be called the Chaotic factor in our experiment and will be denoted accordingly by ' $C h f$ '. We will see why we have called this term the chaotic factor; in fact:

- In case $P_{r}=1$, that is the case of a certain event, then the chaotic factor of the event is equal to 0 .

- In case $P_{r}=0$, that is the case of an impossible event, then $C h f=0$. Hence, in both two last cases, there is no chaos since the outcome is certain and is known in advance.

- In case $P_{r}=0.5, C h f=-0.5$ (Fig. 7).

We notice that:

$$
-0.5 \leq C h f \leq 0
$$

What is interesting here is thus we have quantified both the degree of our knowledge and the chaotic factor of any random event and hence we write now:

$P c^{2}=|Z|^{2}-2 i P_{r} P_{m}=D O K-C h f$

Then we can conclude that:

- $\quad P c^{2}=$ Degree of our knowledge of the systemchaotic factor $=1$, therefore $P c=1$

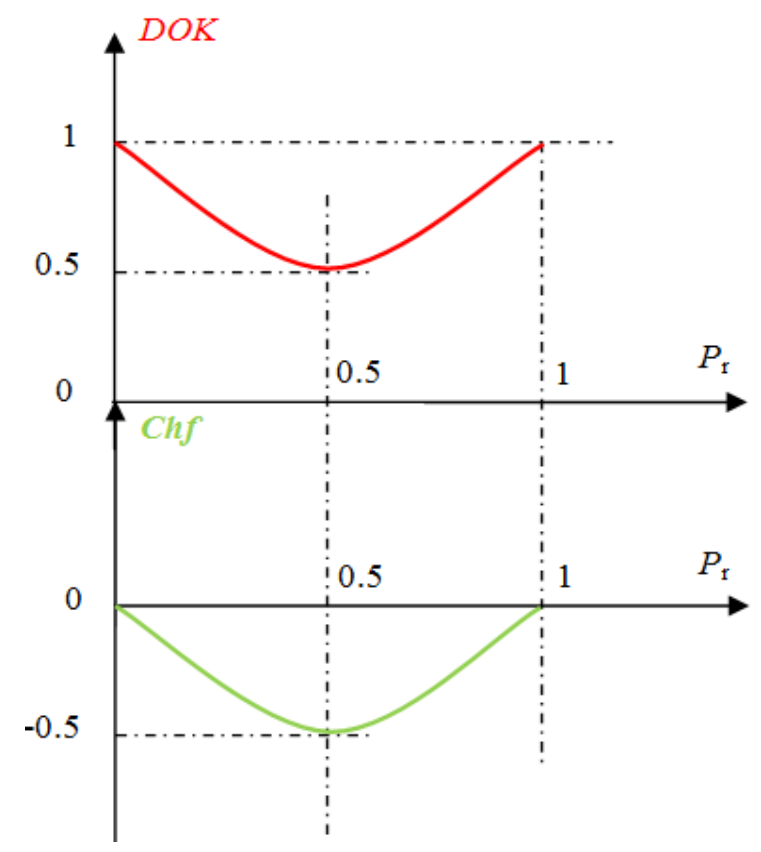

Fig. 7. DOK and Chf 
This directly means that if we succeed to subtract and eliminate the chaotic factor in any random experiment, then the output will always be with the probability $=1$ (Ekeland, 1991; Gleick, 1997; Dalmedico-Dahan and Peiffer, 1986; Gullberg, 1997; SCIENCE et VIE, 1999).

The graph below (Fig. 8) shows the linear relation between both $D O K$ and $C h f$.

To summarize and conclude, as the Degree of Our certain Knowledge or $D O K$ in the real universe $R$ is unfortunately incomplete, the extension to the complex set $\mathrm{C}$ includes the contributions of both the real set of probabilities $R$ and the imaginary set of probabilities $M$. Consequently, this will result in a complete and perfect degree of knowledge in $C=R+M\left(P_{c}=1\right)$. In fact, in order to have a certain prediction of any random event, it is necessary to work in the complex set $C$ in which the chaotic factor is quantified and subtracted from the computed degree of knowledge to lead to a probability in $\mathrm{C}$ equal to one $\left(P c^{2}=D O K-C h f=1\right)$. This hypothesis is verified in four previous research papers by the mean of many examples encompassing both discrete and continuous distributions (Abou Jaoude et al., 2010b; Abou Jaoude, 2013b; 2013c; 2014). The Extended Kolmogorov Axioms (EKA for short) or the complex probability paradigm can be illustrated by the following Fig. 9 .
The Complex Probability Paradigm Applied to Prognostic (Abou Jaoude et al., 2010b; Abou Jaoude, 2013b; 2013c; 2014)

In engineering systems, the remaining useful lifetime prediction is deeply related to many factors that generally have a chaotic and random behavior which decreases the degree of our knowledge of the system.

From the $E K A$, we can deduce that if we add to an event probability in the real set $R$ the imaginary part $M$ (like the lifetime variables) then we can predict the exact probability of the Remaining Useful Lifetime (RUL) with certainty in $C(P c=1)$.

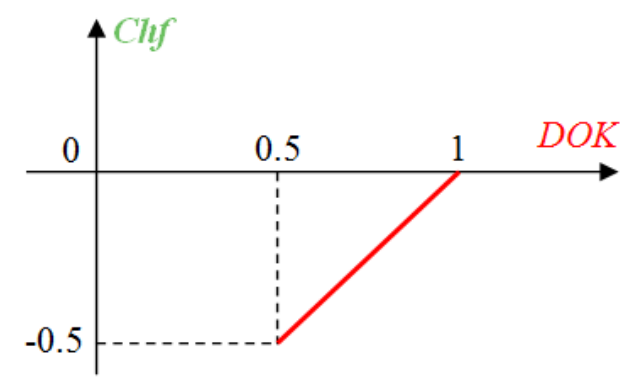

Fig. 8. Graph of $D O K-C h f=1$

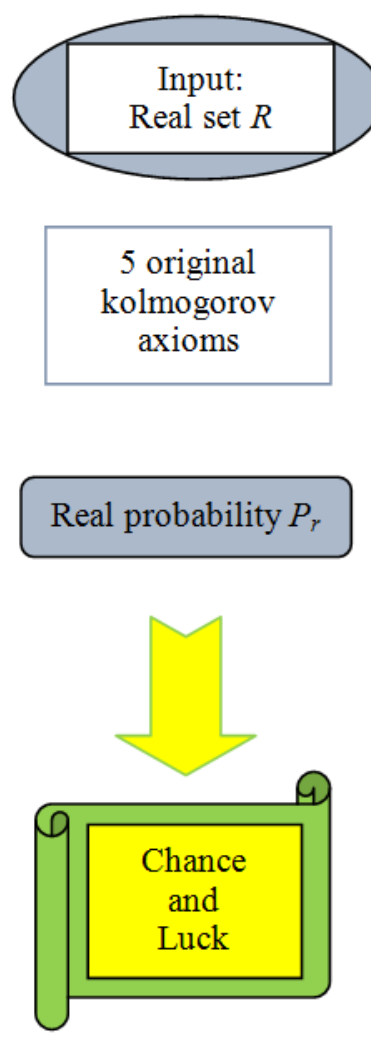

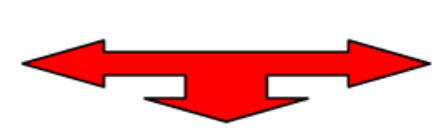

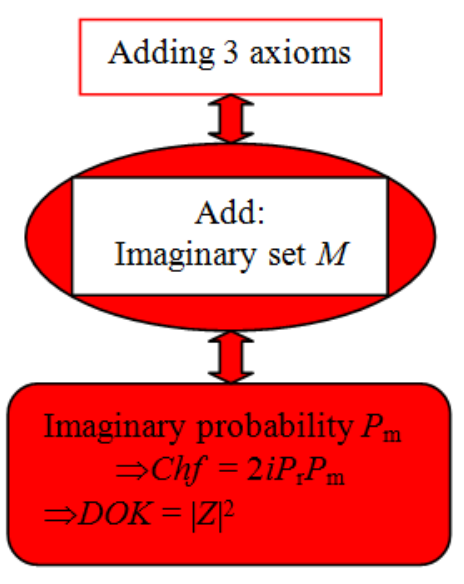

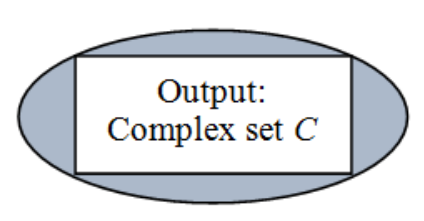

\section{A total of 8 axioms}
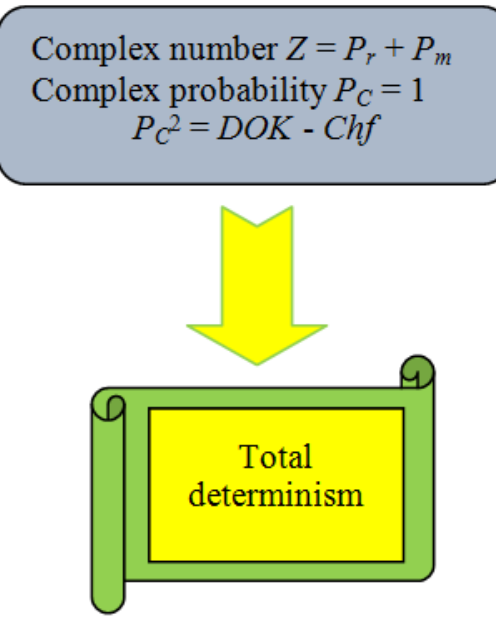

Fig. 9. The EKA or Complex Probability Paradigm 


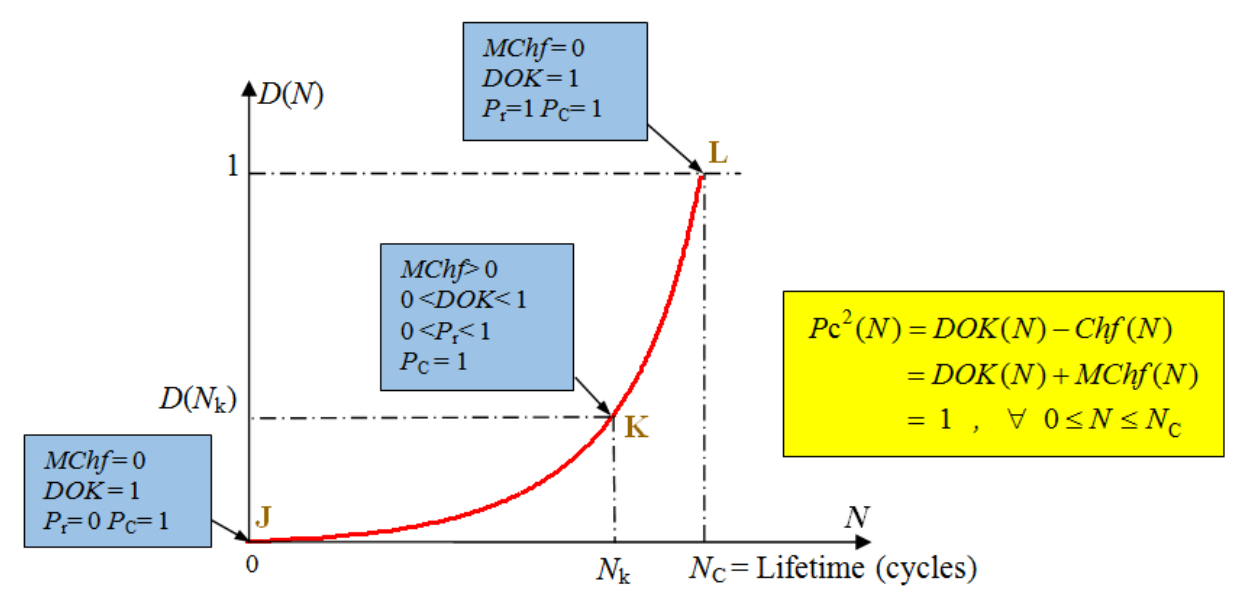

Fig. 10. EKA and the prognostic of degradation

We can apply this idea to prognostic analysis through the degradation evolution of a system. As a matter of fact, prognostic analysis consists in the prediction of the remaining useful lifetime of a system at any instant $t$ or cycle $N$ and during the system functioning.

Let us consider a degradation trajectory $D(N)$ of a system where a specific instant (cycle) $N_{k}$ is studied. The number $N_{k}$ means here the age that is measured by the number of cycles (Fig. 10).

Referring to the (Fig. 11), the previous statement means that at the system age $N_{k}$, the prognostic study must give the prediction of the failure instant $N_{C}$. Therefore, the $R U L$ predicted here at the cycle number $N_{k}$ is the following value:

$\operatorname{RUL}\left(N_{k}\right)=N_{C}-N_{k}$

In fact, at the beginning $\left(N_{k}=0\right)$ (point J), the failure probability $P_{r}=0$, the chaotic factor in our prediction is zero $(C h f=0)$ and our knowledge of the intact system is certain $(D O K=1)$; therefore, $R U L(0)=N_{C}-N_{k}=N_{C}-0=N_{C}$.

If $N_{k}=N_{C}$ (point $L$ ) then $R U L\left(N_{C}\right)=N_{C}-N_{C}=0$ and the failure probability is one $\left(P_{r}=1\right)$. At this point, failure occurs, hence our knowledge of the completely damaged system is certain $(D O K=1)$ and chaos has finished its harmful task so it is no more applicable $(C h f=0)$.

If not (i.e., $0<N_{k}<N_{C}$ ) (point $\mathrm{K}$ ), the probability of the occurrence of this instant and the prediction probability of $R U L$ are both less than one (not certain) due to non-zero chaotic factors. The degree of our knowledge is consequently less than 1 . Thus, by applying here the EKA paradigm, we can determine the system RUL with certainty in $C=R+M$ where $P c=1$ always.

Furthermore, we need in our current study the absolute value of the chaotic factor that will give us the magnitude of the chaotic and random effects on the studied system materialized by the random cycle number $N$ and which lead to a premature system failure. This new term will be denoted accordingly $M C h f$ or Magnitude of the Chaotic factor (Fig. 10). Hence, we can deduce the following:

$$
\begin{aligned}
& \operatorname{MChf}\left(N_{k}\right)=\left|\operatorname{Chf}\left(N_{k}\right)\right| \geq 0 \text { and } \\
& \operatorname{Pc}^{2}\left(N_{k}\right)=\operatorname{DOK}\left(N_{k}\right)-\operatorname{Chf}\left(N_{k}\right) \\
& =\operatorname{DOK}\left(N_{k}\right)+\left|\operatorname{Chf}\left(N_{k}\right)\right|, \text { since }-0.5 \leq \operatorname{Chf}\left(N_{k}\right) \leq 0 \\
& =\operatorname{DOK}\left(N_{k}\right)+\operatorname{MChf}\left(N_{k}\right)=1, \quad \forall 0 \leq N_{k} \leq N_{\mathrm{C}}
\end{aligned}
$$

$\Leftrightarrow 0 \leq \operatorname{MChf}\left(N_{k}\right) \leq 0.5$

where $0.5 \leq \operatorname{DOK}\left(N_{k}\right) \leq 1$

Moreover, we can define two complementary events $E$ and $\bar{E}$ with the respective probabilities:

$$
P_{r o b}(E)=p \text { and } P_{r o b}(\bar{E})=q=1-p
$$

Then $P_{r o b}(E)$ in terms of the cycle number $N_{k}$ is given by:

$$
P_{\mathrm{rob}}(E)=P_{r o b}\left(N \leq N_{k}\right)=F\left(N_{k}\right)
$$

where, $F$ is the Cumulative probability Distribution Function (CDF) of the random variable $N$.

Since $P_{r o b}(E)+P_{r o b}(\bar{E})=1$, therefore, For a cycle $N=$ $N_{k}$ we have:

$$
\begin{aligned}
& P_{r o b}(\bar{E})=1-P_{r o b}(E)=1-P_{r o b}\left(N \leq N_{k}\right) \\
& =P_{r o b}\left(N>N_{k}\right)=1-F\left(N_{k}\right)
\end{aligned}
$$

Let us define the two particular instants:

- $N_{0}=0$ assumed as the initial time of functioning (raw state) corresponding to $D=D_{0} \cong 0$

- $N_{\mathrm{C}}=$ The failure instant (wear out state) corresponding to the degradation $D=1$ 
The boundary conditions are:

- For $N_{0}=0$ then $D=D_{0}$ (initial damage that may be nearly zero) and $F\left(N_{0}\right)=P_{r o b}(N \leq 0)=0$

- For $N=N_{C}$ then $D=1$ and $F(N)=F\left(N_{C}\right)=P_{r o b}\left(N \leq N_{C}\right)=1$

We note also that $F\left(N_{k}\right)$ is a non-decreasing function that varies between 0 and 1 . In fact, $F\left(N_{k}\right)$ is a cumulative probability function. In addition, since $\operatorname{RUL}\left(N_{k}\right)=N_{C}-N_{k}$ and $0 \leq N_{k} \leq N_{C}$ then $\operatorname{RUL}\left(N_{k}\right)$ is a nonincreasing remaining useful lifetime function (Fig. 11).

\section{The New Prognostic Model}

Let us present now the basic assumptions of the new model.

We consider first the cumulative probability distribution function $F(N)$ of the random variable $N$ as equal to the degradation function itself, that means:

$$
F\left(N_{k}\right)=P_{r o b}\left(N \leq N_{k}\right)=\sum_{N=0}^{N=N_{k}} P_{r o b}(N)=D\left(N_{k}\right)
$$

We note that we are dealing here with discrete random functions depending on the discrete random number of load cycles.

This basic assumption is plausible since:

- $\quad$ Both $F$ and $D$ are non-decreasing functions

- Both are cumulative functions starting from 0 and ending with 1

- Both functions are without measure units: $F$ is an index quantifying chance and randomness, as well as $D$ which is an index quantifying degradation and system damage

Then, we assume that the real probability $P_{r}(N)$ is equal to (Fig. 11 and 12):

$$
\begin{aligned}
& P_{r}\left(N_{\mathrm{k}}\right)=\psi_{j} \times\left[P_{r o b}\left(N \leq N_{\mathrm{k}}\right)-P_{r o b}\left(N \leq N_{k-1}\right)\right] \\
& =\psi_{j} \times\left[F\left(N_{k}\right)-F\left(N_{k-1}\right)\right] \\
& =\psi_{j} \times\left[\sum_{N=0}^{N=N_{\mathrm{k}}} P_{r o b}(N)-\sum_{N=0}^{N=N_{k-1}} P_{r o b}(N)\right] \\
& =\psi_{j} \times\left[D\left(N_{k}\right)-D\left(N_{k-1}\right)\right] \\
& =\psi_{j} \text { times the jump in } F(N) \text { or } D(N) \text { from } \\
& N=N_{\mathrm{k}-1} \text { to } N=N_{\mathrm{k}}
\end{aligned}
$$

where, $\quad N=0,1,2, \ldots, N_{k-1}, N_{\mathrm{k}} N_{k+1}, \ldots N_{C}=$ the number of load cycles and $N_{0}=0=$ the initial number of cycles at the simulation beginning. It corresponds to a degradation $D=D\left(N_{0}\right)=D_{0}$ which is generally considered to be nearly equal to 0 ; hence it follows that, $F\left(N_{0}\right)$ is logically equal to 0 just like any cumulative probability distribution function:

- $\quad N_{1}=$ the first load cycle

- $\quad \ldots \ldots \ldots$

- $\quad N_{k}=$ the kth load cycle

- $\quad \ldots \ldots \ldots$

- $\quad N_{C}=$ The number of cycles till system failure $=$ the critical number of load cycles. It corresponds to $D=$ $D_{\mathrm{C}}=1$. It follows directly that $F\left(N_{\mathrm{C}}\right)=D_{\mathrm{C}}=1$

- $\psi_{j}=$ The simulation factor that depends on the road profile

It is $\psi_{1}=107.41$ for the first road profile (severe, $j=1$ ), it is $\psi_{2}=155.04$ for the second road profile (fair, $j=2$ ), it is $\psi_{3}=301.20$ for the third road profile (good, $j=3$ ).

We can observe that $P_{\mathrm{r}}(N)$ is a discrete step function, just like any discrete CDF, where the amount of the jump is a function of the degradation and damage evolution (Fig. 12 and 13). Hence, it measures the probability of the system failure. Consequently, we have linked here probability theory to degradation measure.

Initially we have:

$$
\begin{aligned}
& P_{r}\left(N_{k}=N_{0}=0\right)=\psi_{j} \times F\left(N_{0}\right)=\psi_{j} \times D\left(N_{0}\right) \\
& =\psi_{j} \times 0=0
\end{aligned}
$$

Moreover:

$P_{r}\left(N_{k}\right)=\psi_{j} \times f_{j}\left(N_{k}\right)$

where, $f_{j}\left(N_{k}\right)$ is the Probability Density Function (PDF). Knowing that, from classical probability theory, we have always:

$$
\sum_{N_{k}=0}^{N_{k}=N_{\mathrm{c}}} f_{\mathrm{j}}\left(N_{k}\right)=1, \text { for } j=1,2,3
$$

That means for any road profile.

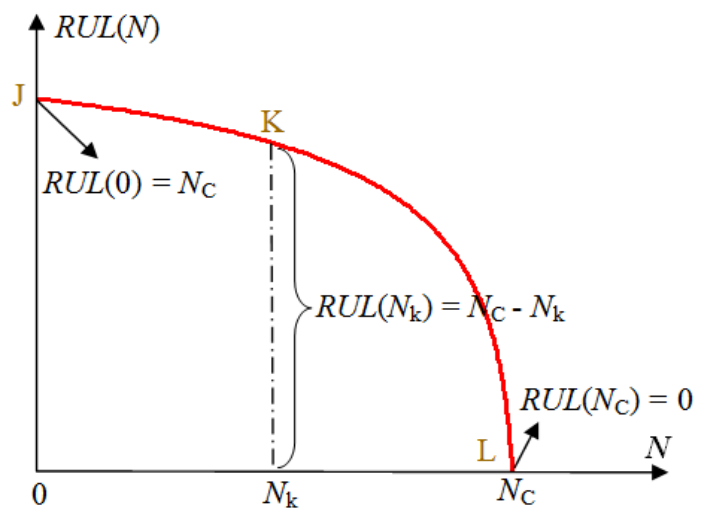

Fig. 11. RUL prognostic model 


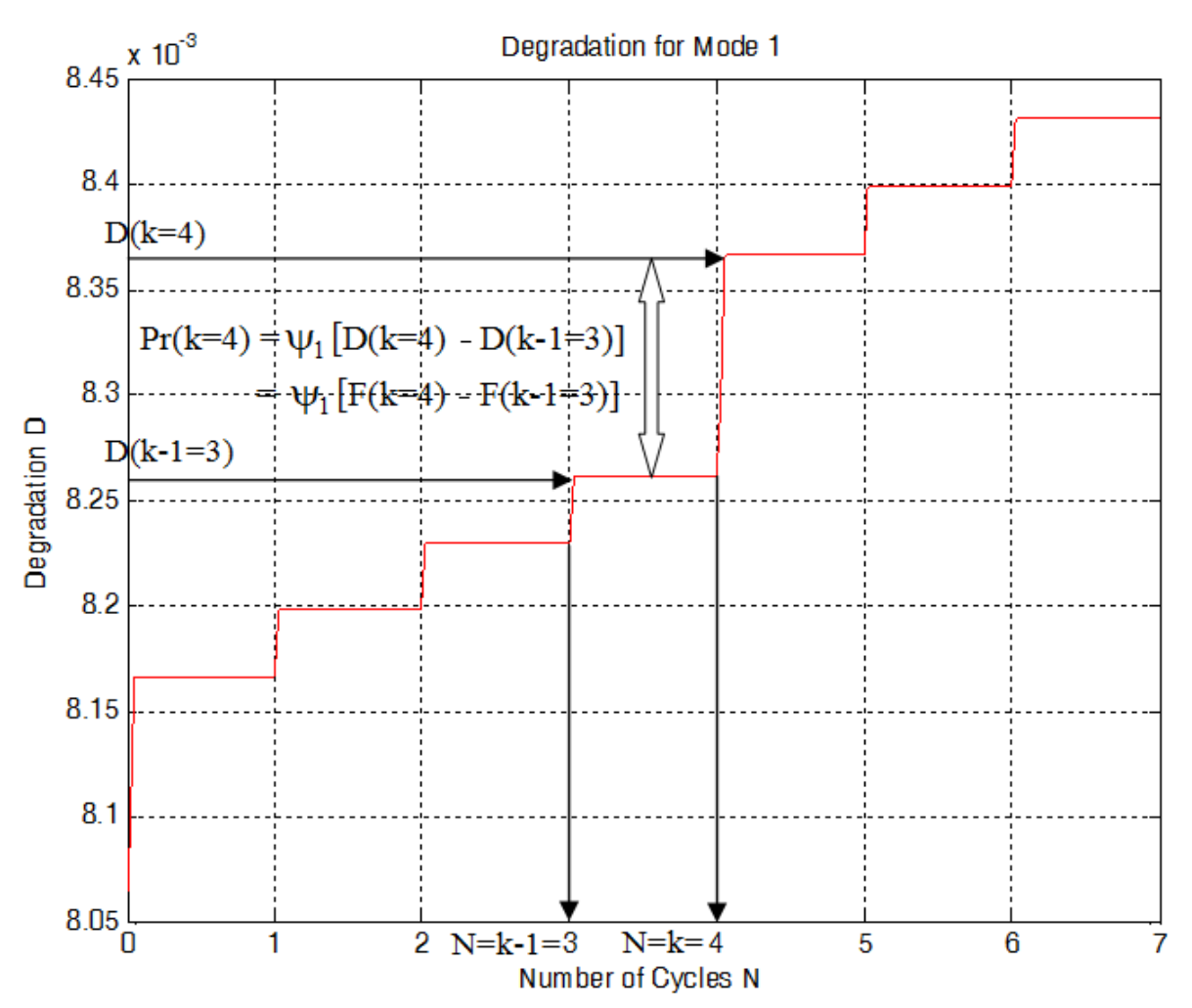

Fig. 12. $P r$, degradation and the CDF step functions

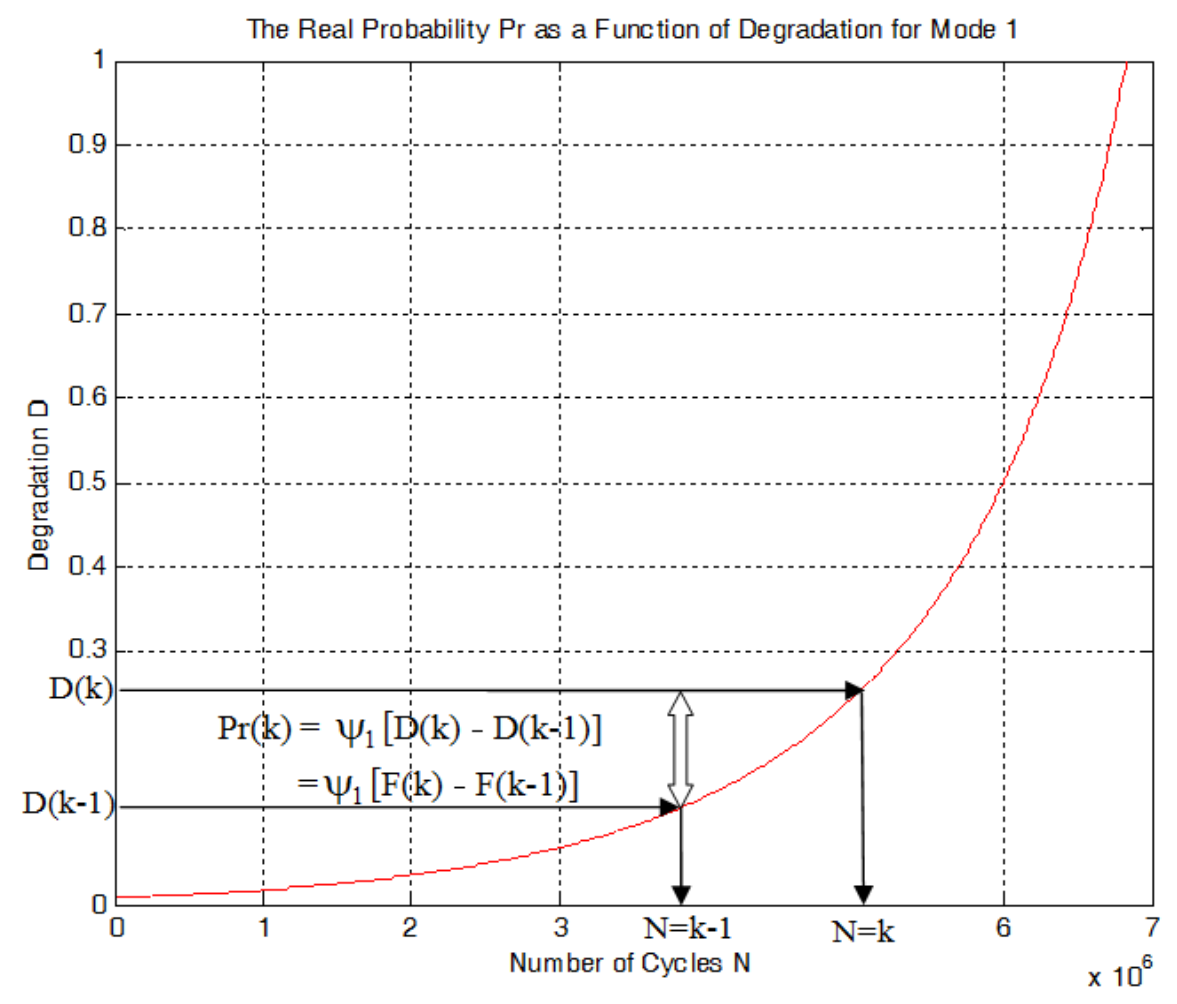

Fig. 13. $P_{r}$ function of degradation 
Therefore, we can deduce that:

$$
\begin{aligned}
& \sum_{N_{k}=0}^{N_{k}=N_{c}} P_{r}\left(N_{k}\right)=\psi_{j} \times \sum_{N=0}^{N=N_{c}} P_{r o b}(N) \\
& =\psi_{j} \times P_{r o b}\left(0 \leq N \leq N_{C}\right) \\
& =\psi_{j} \times\left[F\left(N=N_{C}\right)-F\left(N=N_{0}\right)\right] \\
& =\psi_{j} \times\left[D\left(N_{C}\right)-D\left(N_{0}\right)\right] \\
& =\psi_{j} \times F\left(N_{C}\right)=\psi_{j} \times D\left(N_{C}\right)=\psi_{j} \times 1 \\
& =\psi_{j} \times \sum_{N_{k}=0}^{N_{k}=N_{c}} f_{j}\left(N_{k}\right)=\psi_{j}, \text { for } j=1,2,3
\end{aligned}
$$

This is reasonable since $P_{r}\left(N_{k}\right)$ here is a cumulative probability function (Fig. 14) Notice that:

$$
\begin{aligned}
& 0 \leq P_{r}\left(N_{k}\right) \leq 1,0 \leq F\left(N_{k}\right) \leq 1 \\
& \text { and }\left(D_{0} \cong 0\right) \leq D\left(N_{k}\right) \leq\left(D_{C}=1\right) \\
& \text { for every } 0 \leq N_{k} \leq N_{C}
\end{aligned}
$$

And:

$$
\begin{aligned}
& \text { If } N_{k} \rightarrow 0 \Rightarrow \\
& D \rightarrow D_{0} \cong 0 \Rightarrow F \rightarrow 0 \Rightarrow P_{r}\left(N_{k}\right) \rightarrow 0 \\
& \text { If } N_{k} \rightarrow N_{C} \Rightarrow \\
& D \rightarrow D_{C}=1 \Rightarrow F \rightarrow 1 \Rightarrow P_{r}\left(N_{k}\right) \rightarrow 1
\end{aligned}
$$

This, since the degradation is very flat near 0 and starts increasing with $N$, becoming very acute at $N=N_{\mathrm{C}}$; hence, near $N_{\mathrm{C}}, P_{\mathrm{r}}$ is the greatest and is nearly equal to 1 .

Furthermore, we have:

- $\operatorname{RUL}\left(N_{k}\right)=N_{C}-N_{k}$ and it corresponds to a degradation $D\left(N_{k}\right)$

- $\operatorname{RUL}\left(N_{\mathrm{k}-1}\right)=N_{C}-N_{k-1}$ and it corresponds to a degradation $D\left(N_{k-1}\right)$, this implies that (Fig. 15):

$$
\begin{aligned}
& P_{r}\left(N_{k}\right)=\psi_{j} \times\left[D\left(N_{k}\right)-D\left(N_{k-1}\right)\right] \\
& =\psi_{j} \times\left\{D\left[N_{C}-\operatorname{RUL}\left(N_{k}\right)\right]-D\left[N_{C}-\operatorname{RUL}\left(N_{k-1}\right)\right]\right\}
\end{aligned}
$$

\section{Analysis and Extreme Chaotic Conditions}

Although (Abou Jaoude, 2015) but there are random factors (temperature, humidity, applied load location, water action, etc...) that affect the system and make its degradation function deviate from its calculated trajectory predefined by these laws. An updated follow-up of the degradation behavior with time or cycle number is done by $P_{\mathrm{r}}$ due to its definition that evaluates the jumps in $D$.

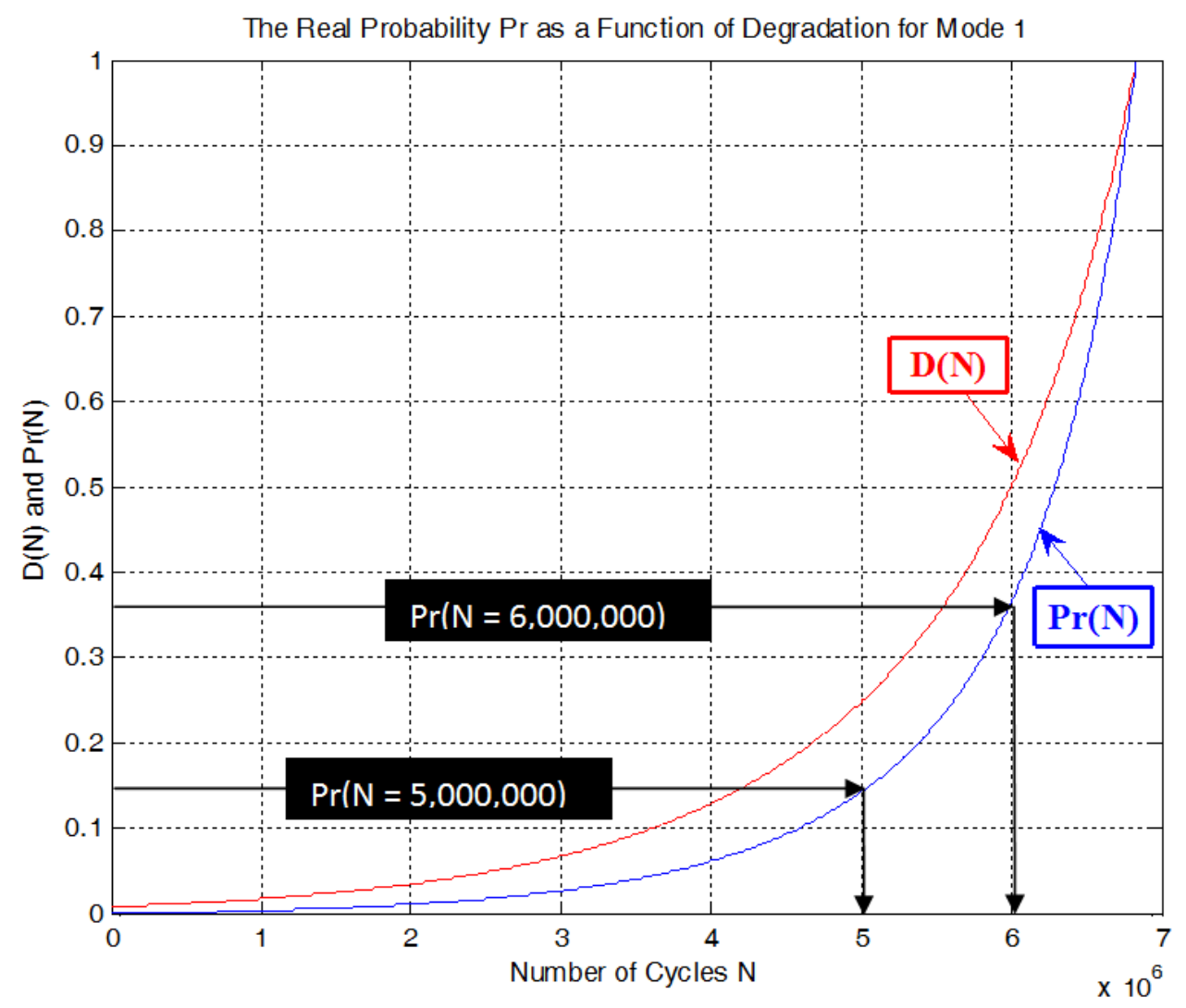

Fig. 14. Degradation and $P_{r}$ 


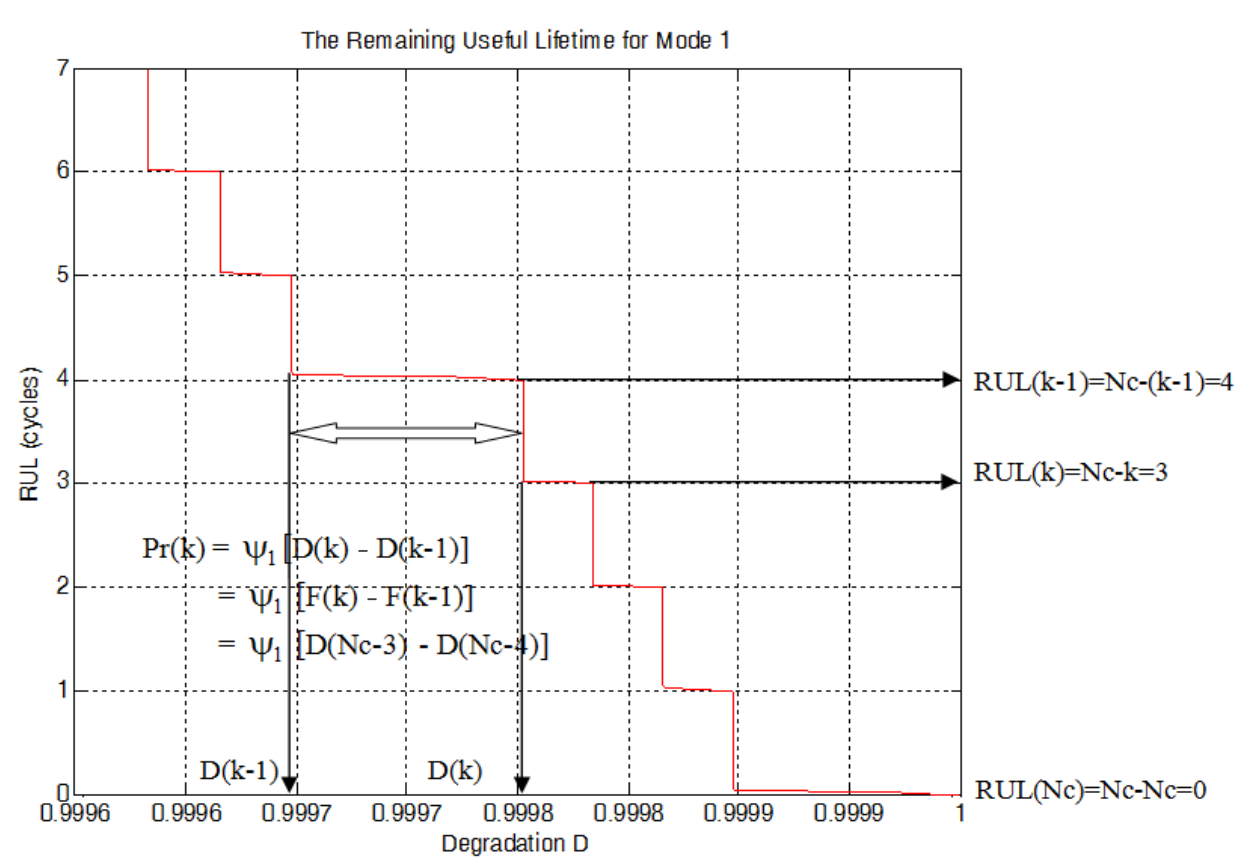

Fig. 15. Pr and RUL

In fact, an accentuated effect of chaos on the system leads to a bigger jump in the degradation trajectory and hence to a greater probability of failure $P_{r}$. If for example, due to extreme chaotic causes, $D$ jumps directly from 0 to 1 then $R U L$ goes straight from $N_{\mathrm{C}}$ to 0 and consequently $P_{r}$ jumps instantly from 0 to $\psi_{j}$ :

$$
\begin{aligned}
& P_{r}\left(N_{\mathrm{k}}\right)=\psi_{j} \times\left[D\left(N_{k}\right)-D\left(N_{k-1}\right)\right] \\
& =\psi_{j} \times\left[D\left(N_{C}\right)-D\left(N_{0}\right)\right] \\
& =\psi_{j} \times[1-0]=\psi_{j} \\
& =\sum_{N_{k}=0}^{N_{k}=N_{\mathrm{c}}} P_{\mathrm{r}}\left(N_{k}\right)=P_{r}\left(N_{0}\right)+P_{r}\left(N_{C}\right) \\
& =0+\psi_{j} \times(1)
\end{aligned}
$$

where, $N$ goes directly from $N_{0}$ to $N_{C}$.

In the ideal extreme case, if the system never deteriorates staying indefinitely at $D_{0}=0$ then $R U L$ remains equal to $N_{\mathrm{C}}$ and accordingly the jump in $D$ is always 0 , so ideally the probability of failure stays 0 :

$$
\begin{aligned}
& P_{r}\left(N_{\mathrm{k}}\right)=\psi_{j} \times\left[D\left(N_{k}\right)-D\left(N_{k-1}\right)\right] \\
& =\psi_{j} \times\left[D\left(N_{0}\right)-D\left(N_{0}\right)\right] \\
& =\psi_{j} \times[0-0]=0,
\end{aligned}
$$

Where:

$$
\mathrm{D}\left(N_{0}\right)=D\left(N_{1}\right)=\ldots=D\left(N_{k-1}\right)=D\left(N_{k}\right)=\ldots \ldots=0
$$

The Evaluation of the Paradigm Parameters

We can infer from what has been elaborated previously the following:

The real probability:

$P_{r}\left(N_{\mathrm{k}}\right)=\psi_{j} \times\left[D\left(N_{k}\right)-D\left(N_{k-1}\right)\right], j=1,2,3$

The imaginary probability:

$\mathrm{P}_{m}\left(N_{k}\right)=i\left[1-P_{r}\left(N_{k}\right)\right]=$
$i\left\{1-\psi_{j} \times\left[D\left(N_{k}\right)-D\left(N_{k-1}\right)\right]\right\}$

The complementary probability:

$\mathrm{P}_{m}\left(N_{k}\right) / i=1-P_{r}\left(N_{k}\right)=1-\psi_{j} \times\left[D\left(N_{k}\right)-D\left(N_{k-1}\right)\right]$

The complex probability vector:

$Z\left(N_{k}\right)=P_{r}\left(N_{k}\right)+P_{m}\left(N_{k}\right)=P_{r}\left(N_{k}\right)+i\left[1-P_{r}\left(N_{k}\right)\right]$

The degree of our knowledge:

$$
\begin{aligned}
& D O K\left(N_{k}\right)=\left|Z\left(N_{k}\right)\right|^{2} 1+2 i P_{r}\left(N_{k}\right) P_{m}\left(N_{k}\right) \\
& =1-2 P_{r}\left(N_{k}\right)\left[1-P_{r}\left(N_{k}\right)\right]=1-2 P_{r}\left(N_{k}\right)+2 P_{r}^{2}\left(N_{k}\right)
\end{aligned}
$$

The chaotic factor: 


$$
\begin{aligned}
& \operatorname{Chf}\left(N_{k}\right)=2 i P_{r}\left(N_{k}\right) P_{\mathrm{m}}\left(N_{k}\right) \\
& =-2 P_{r}\left(N_{k}\right)\left[1-P_{r}\left(N_{\mathrm{k}}\right)\right]=-2 P_{r}\left(N_{k}\right)+2 P_{r}^{2}\left(N_{k}\right)
\end{aligned}
$$

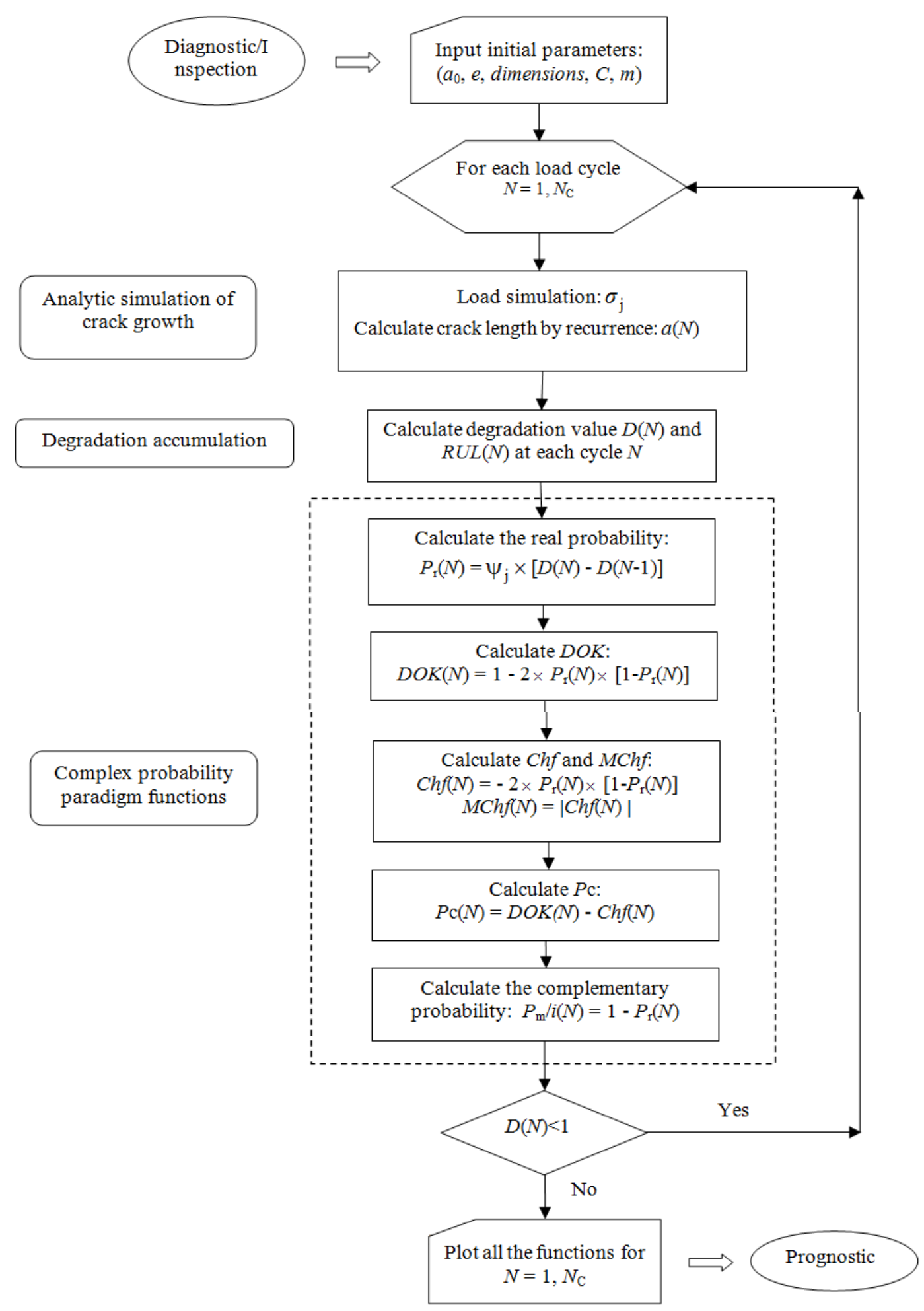

Fig. 16. Flowchart of the complex probability prognostic model

Chf is null when $P_{\mathrm{r}}\left(N_{\mathrm{k}}\right)=P_{\mathrm{r}}(0)=0$ (point $\mathrm{J}$ ) and when $P_{\mathrm{r}}\left(N_{\mathrm{k}}\right)=P_{\mathrm{r}}\left(N_{\mathrm{C}}\right)=1$ (point L) (Fig. 10 and 11). 
The Magnitude of the Chaotic Factor MChf:

$$
\begin{aligned}
& \operatorname{MChf}\left(N_{k}\right)=\left|\operatorname{Chf}\left(N_{k}\right)\right|=2 P_{r}\left(N_{k}\right)\left[1-P_{r}\left(N_{k}\right)\right] \\
& =2 P_{r}\left(N_{k}\right)-2 P_{r}^{2}\left(N_{k}\right)
\end{aligned}
$$

MChf is null when $P_{\mathrm{r}}\left(N_{\mathrm{k}}\right)=P_{\mathrm{r}}(0)=0$ (point $\left.\mathrm{J}\right)$ and when $P_{\mathrm{r}}\left(N_{\mathrm{k}}\right)=P_{\mathrm{r}}\left(N_{\mathrm{C}}\right)=1$ (point L) (Fig. 10 and 11).

At any instant $N_{\mathrm{k}}$ (point K) (Fig. 10 and 11), the probability expressed in the complex set $C$ is:

$$
\begin{aligned}
& P c\left(N_{k}\right)^{2}=\left[P_{r}\left(N_{k}\right)+P_{m}\left(N_{k}\right) / i\right]^{2} \\
& =\left|Z\left(N_{k}\right)\right|^{2}-2 i P_{r}\left(N_{k}\right) P_{m}\left(N_{k}\right) \\
& =\operatorname{DOK}\left(N_{k}\right)-\operatorname{Chf}\left(N_{k}\right)=1
\end{aligned}
$$

Then:

$$
\begin{aligned}
& P c\left(N_{\mathrm{k}}\right)=P_{r}\left(N_{k}\right)+P_{m}\left(N_{k}\right) / i=P_{r}\left(N_{k}\right) \\
& +\left[1-P_{r}\left(N_{k}\right)\right]=1 \text { always }
\end{aligned}
$$

Hence, the prediction of $D\left(N_{k}\right)$ and $\operatorname{RUL}\left(N_{k}\right)$ of the system in $C$ is permanently certain.
Let us consider thereafter the suspension system to model the cumulative distribution function $F\left(N_{k}\right)=D\left(N_{k}\right)$ and visualize as well as quantify all the $E K A$ parameters.

Flowchart of the Complex Probability Prognostic Model

The following flowchart summarizes all the procedures of the proposed complex probability prognostic model Fig. 16.

\section{Simulation of the New Paradigm}

The Parameters Analysis in the Suspension Prognostic for Mode 1

We rescaled $R U L=[0 ; 6,836,000]$ to $[0 ; 1]$ in order to fit and represent it with all the $E K A$ parameters and $D$ which vary in $[0 ; 1]$ on the same graph while having all of them as functions of the number of cycles $N=[0 ; 6,836,000]$.

We notice from the Fig. $17-20$ that the $D O K$ is maximum $(D O K=1)$ when $M C h f$ is minimum (MChf $=0$ ) (points $J$ and $L$ ) and that means when the magnitude of the chaotic factor $(M C h f)$ decreases our certain knowledge $(D O K)$ increases.

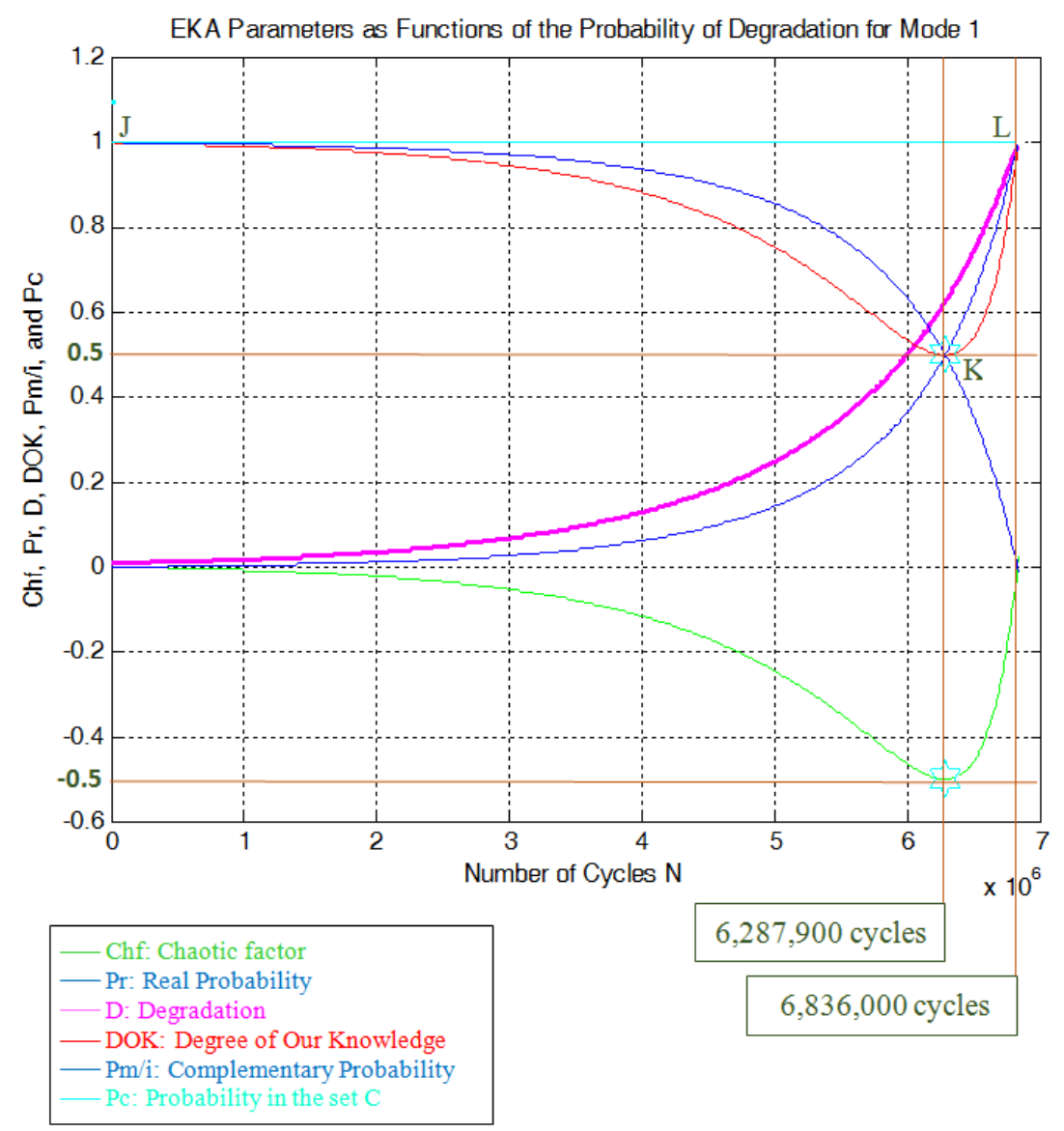

Fig. 17. Degradation and $E K A$ parameter for mode 1 


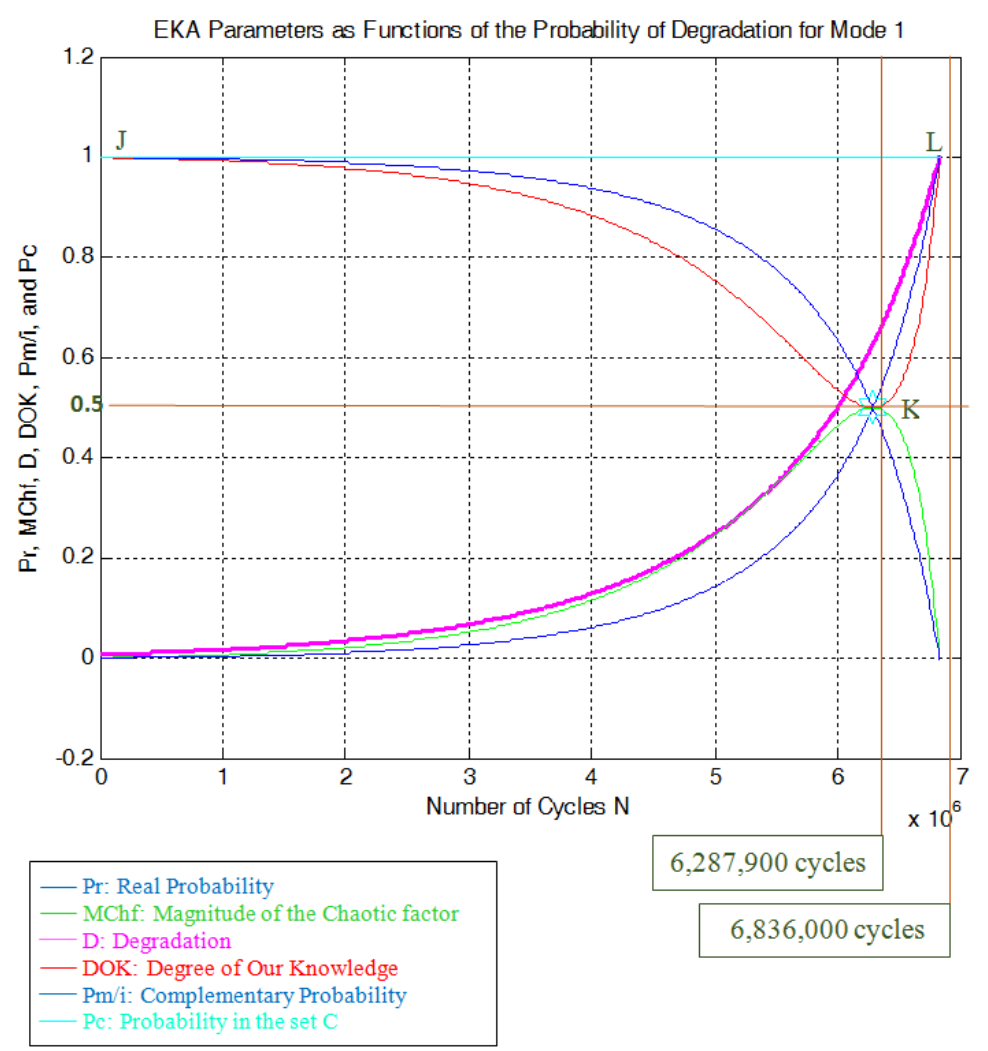

Fig. 18. Degradation and EKA parameters with MChf for mode 1

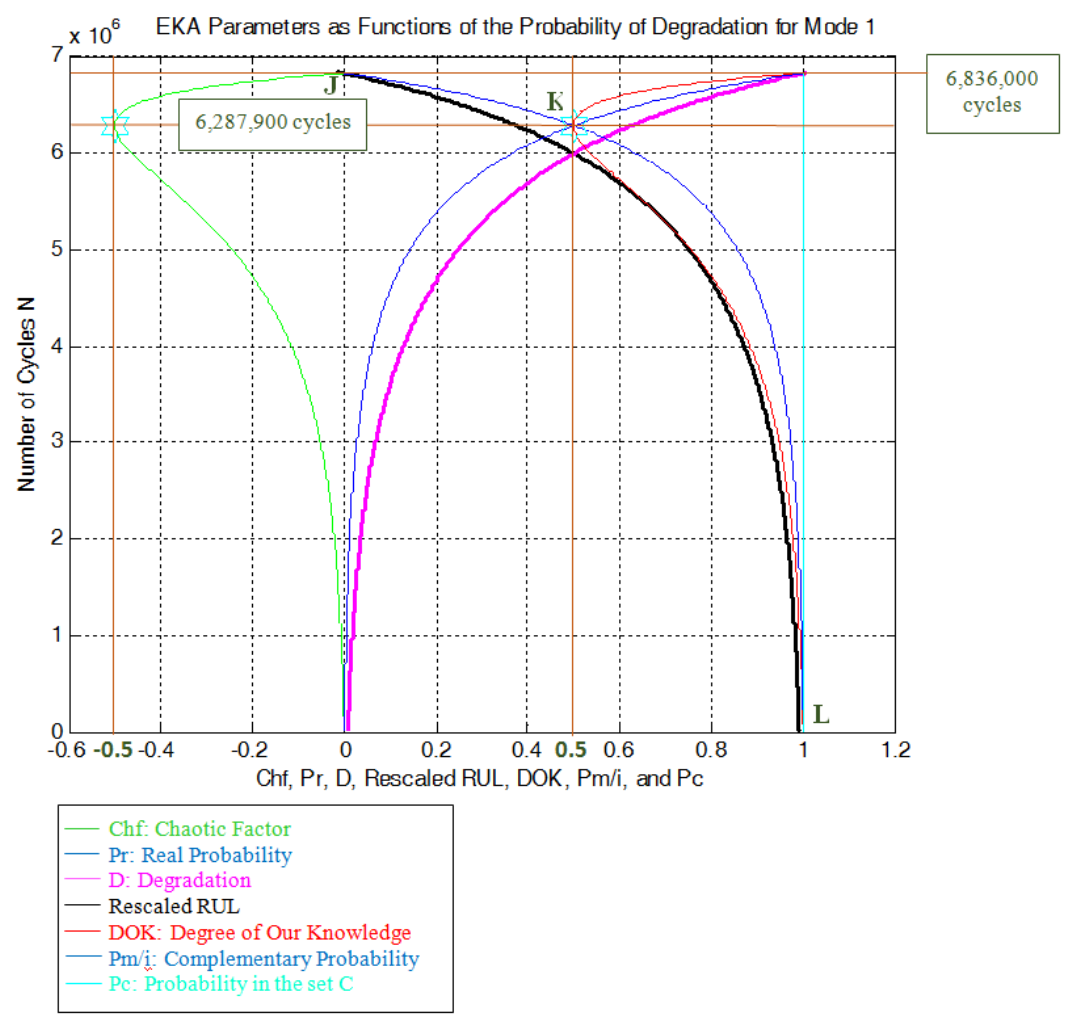

Fig. 19. Degradation, rescaled $R U L$ and $E K A$ parameters for mode 1 


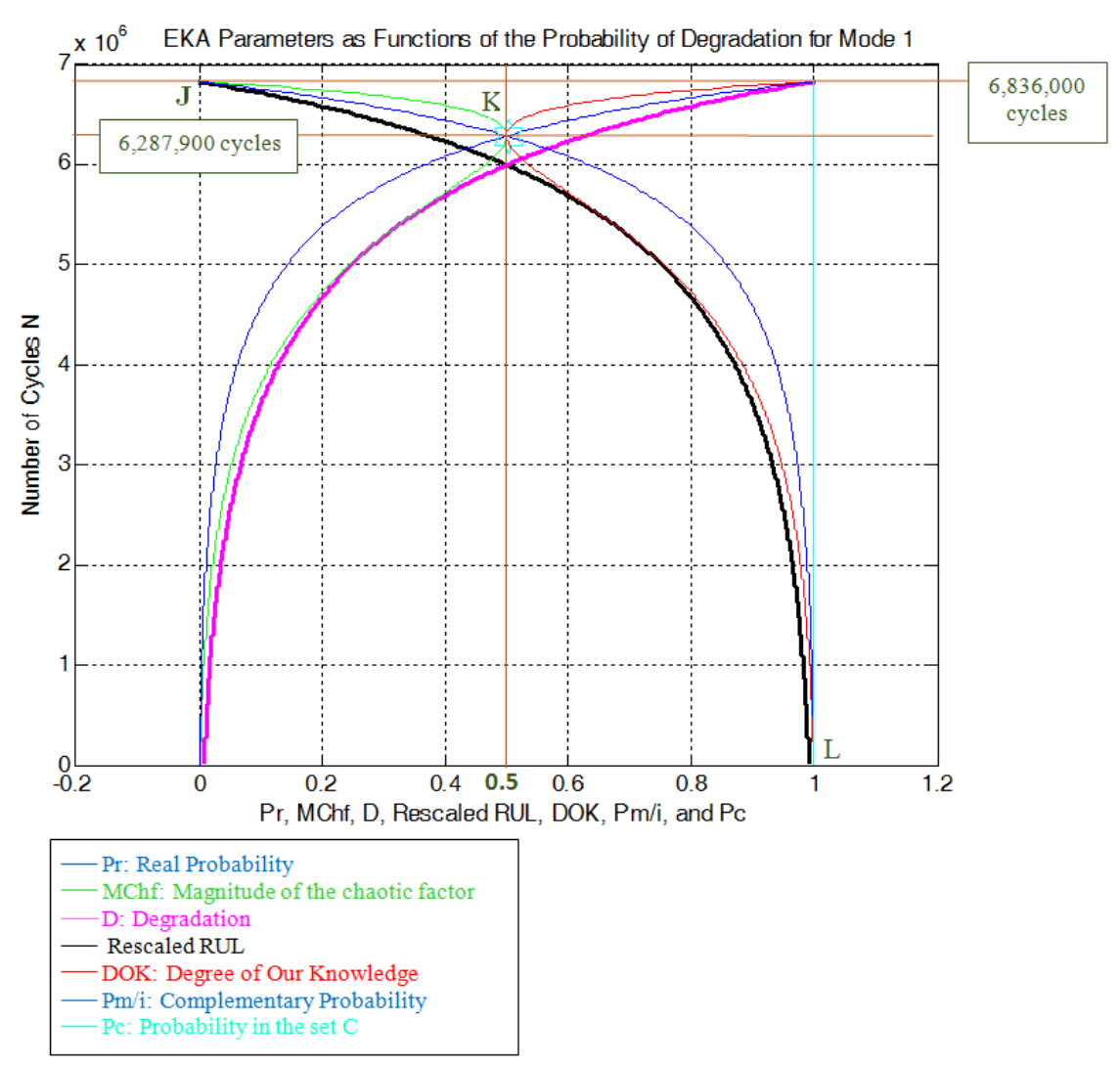

Fig. 20. Degradation, rescaled $R U L$ and $E K A$ parameters with $M C h f$ for mode 1

At the beginning (point $\mathrm{J}) P_{r}\left(N=N_{0}=0\right)=0$, the system is intact (zero damage: $D=0$ ) and has zero chaotic factor $(\operatorname{Chf}(0)=\operatorname{MChf}(0)=0)$ before any usage, at this instant (cycles number) $D O K(0)=1$ and $R U L(0)=$ $N_{C}-0=N_{\mathrm{C}}=6,836,000$ cycles $=N_{C} / 1$. We have here the probability of the system collapse $P_{r}(0)$ is 0 ; hence, this is the system raw state. At this point $P_{m} / i(0)=1$, thus $P_{C}(0)=P_{r}(0)+P_{m} / i(0)=0+1=1$.

Afterward, $0<N<N_{C}, R U L(N)=N_{C}-N$ with $P_{\mathrm{r}}(N)=$ $\psi_{1} \times[D(N)-D(N-1)] \neq 0$ and $P c(N)=P_{r}(N)+P_{m} / i(N)=1$ and $M C h f$ starts to increase during the functioning due to the environment and intrinsic conditions thus leading to a decrease in $D O K$.

If $N=N_{C} / 2=6,836,000 / 2=3,418,000$ cycles $=$ half life of the suspension system, then $R U L=N_{C}-N / 2=$ $3,418,000$ cycles $=N_{\mathrm{C}} / 2, D=0.0887, D O K=0.9265$, Chf $=-0.0735, M C h f=0.0735, P_{\mathrm{r}}=0.0382, P_{\mathrm{m}} / i=$ 0.9618 , with $P c=P_{r}+P_{m} / i=0.0382+0.9618=1$.

If $D=0.5$ then $N=9,573,100$ cycles, $R U L=N C-N=$ $1,276,900$ cycles $=N C / 8.5, D O K=0.5403, C h f=-$ $0.4597, M C h f=0.4597, P_{r}=0.3581, P_{m} / i=0.6419$, with $P c=P_{r}+P_{m} / i=0.3581+0.6419=1$. At this point, both the rescaled $R U L$ and $D$ intersect. We can see that with the increase of $N$ and hence the decrease of $R U L$, the probability of failure $P_{r}$ increases also. Furthermore, notice in the last Fig. 20 the complete symmetry at the vertical axis $D=1 / 2=$ degradation half way to complete damage.

If $N=6,287,900$ cycles (point $\mathrm{K}$ ) both $D O K$ (minimum) and $M C h f$ (maximum) reach 0.5 where $R U L(N)=N_{C}-N=6,836,000-6,287,900=548,100$ cycles $=N_{C} / 12.47, D=0.6274, P_{r}=0.5, P_{m} / i=0.5$ and $C h f=-0.5$, with $P_{c}=P_{r}+P_{m} / i=0.5+0.5=1$ as always. Thus, all the $E K A$ parameters will intersect at the point $\mathrm{K}$. We have here maximum chaos and the minimum of the system knowledge; therefore, the probability of the system crash is $P_{r}=1 / 2=$ probability half way to complete damage.

If $D=0.9$ then $N=6,718,100$ cycles, $R U L=N_{C}-N=$ 117,900 cycles $=N_{C} / 58, D O K=0.7550, C h f=-0.2450$, $M C h f=0.2450, P_{r}=0.8571, P_{m} / i=0.1429, P c=P_{r}+$ $P_{m} / i=0.8571+0.1429=1$. Here, since $D=0.9$ which is very close to 1 , then the failure probability $P_{r}$ is very near to 1 since we will reach total damage very soon.

With the increase of the time of functioning, MChf and $C h f$ return to zero, $D O K$ returns to 1 where we reach total damage $(D=1)$ at $N=N_{C}=6,836,000$ cycles and hence the breakdown of the system (point L). At this last point, failure here is certain, this is the system wear out state; therefore, $P_{r}=1, P_{m} / i=0, R U L(N)=N_{C}-N=N_{C}-N_{C}=0$ with $P c=P_{r}+P_{m} / i=1+0=1$ and so the logical explanation of the value of $D O K=1$ follows. 
We note that the same logic and analysis concerning the degradation, the remaining useful lifetime, as well as all the $E K A$ parameters, apply for all the three modes of roads.

\section{The Complex Probability Cubes}

In the first figure (Fig. 21), we can see the simulation of DOK and Chf as functions of each other and of the cycles number $N$ for mode 1 of roads. The line in magenta is $P c^{2}(N)=D O K(N)-C h f(N)=1=P c(N)$. This line, projected on the $N=0$ plane, starts at the point $(D O K=1, C h f=0)$ when $N=0$, reaches the point $(D O K$ $=0.5, C h f=-0.5$ ) when $N=6,287,900$ cycles (point $\mathrm{K}$ in Fig. 17-20) and returns at the end to $(D O K=1, C h f=0)$ when $N=N_{C}=6,836,000$ cycles. The other curves are the graphs of $\operatorname{DOK}(N)$ and $\operatorname{Chf}(N)$ in different planes. Notice that they all have a minimum at $N=6,287,900$ cycles (point K in Fig. 17-20), as explained previously.

In the second figure (Fig. 22), we can notice the simulation of the failure probability $P_{r}(N)$ and its complementary probability $P_{m} / i(N)$ in terms of the cycles number $N$ for mode 1 of roads. The line in cyan is $P c^{2}(N)$ $=P_{r}(N)+P_{m} / i(N)=1=P c(N)$. This line, projected on the $N=0$ plane, starts at the point $\left(P_{r}=0, P_{m} / i=1\right)$ and ends at the point $\left(P_{r}=1, P_{m} / i=0\right)$. The red curve represents
$P_{r}(N)$ in the plane $P_{r}=P_{m} / i$ and the blue curve represents $P_{m} / i(N)$ in the plane $P_{r}+P_{m} / i=1$. Notice the importance of the point $\left(P_{r}=0.5, P_{m} / i=0.5\right)$ corresponding to $N=$ $6,287,900$ cycles (point $\mathrm{K}$ in Fig. 17-20). Note that similar cubes can be drawn for modes 2 and 3 with their corresponding $N_{\mathrm{C}}$ and points $\mathrm{J}, \mathrm{K}$ and $\mathrm{L}$.

\section{The Parameters Analysis in the Suspension Prognostic for Mode 2}

Just like for mode 1 simulations, we rescaled $R U L=$ $[0 ; 10,850,000]$ to $[0 ; 1]$ in order to fit and represent it with all the $E K A$ parameters and $D$ which vary in $[0 ; 1]$ on the same graph while having all of them as functions of the number of cycles $N=[0 ; 10,850,000]$.

We note from the Fig. 23-26 that the $D O K$ is maximum $(D O K=1)$ when $M C h f$ is minimum $(M C h f=0)$ (points $\mathbf{J}$ and $\mathrm{L}$ ) and that means when the magnitude of the chaotic factor $(M C h f)$ decreases our certain knowledge increases.

At the beginning (point J) $P_{r}(N=0)=0$, the system is intact (zero damage: $D=0$ ) and has zero chaotic factor $(\operatorname{Chf}(0)=M \operatorname{Chf}(0)=0)$ before any usage. At this instant (cycles number) $D O K(0)=1$ and $R U L(0)=N_{C}-0=N_{C}=$ $10,850,000$ cycles $=N_{C} / 1$. Here $P_{m} / i(0)=1$, with $P c(0)$ $=P_{r}(0)+P_{m} / i(0)=0+1=1$.
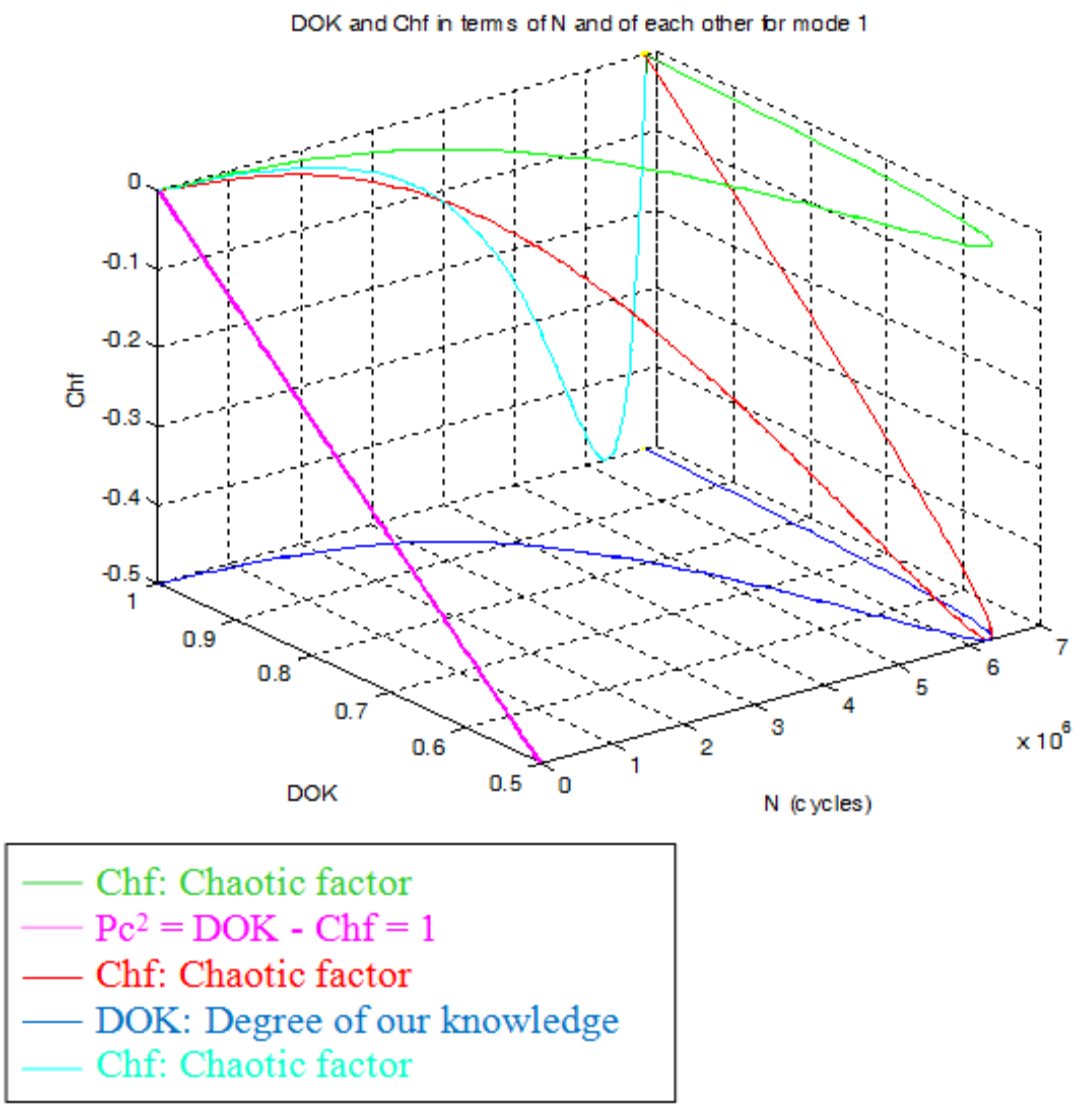

Fig. 21. DOK and Chf in terms of $N$ and of each other for mode 1 


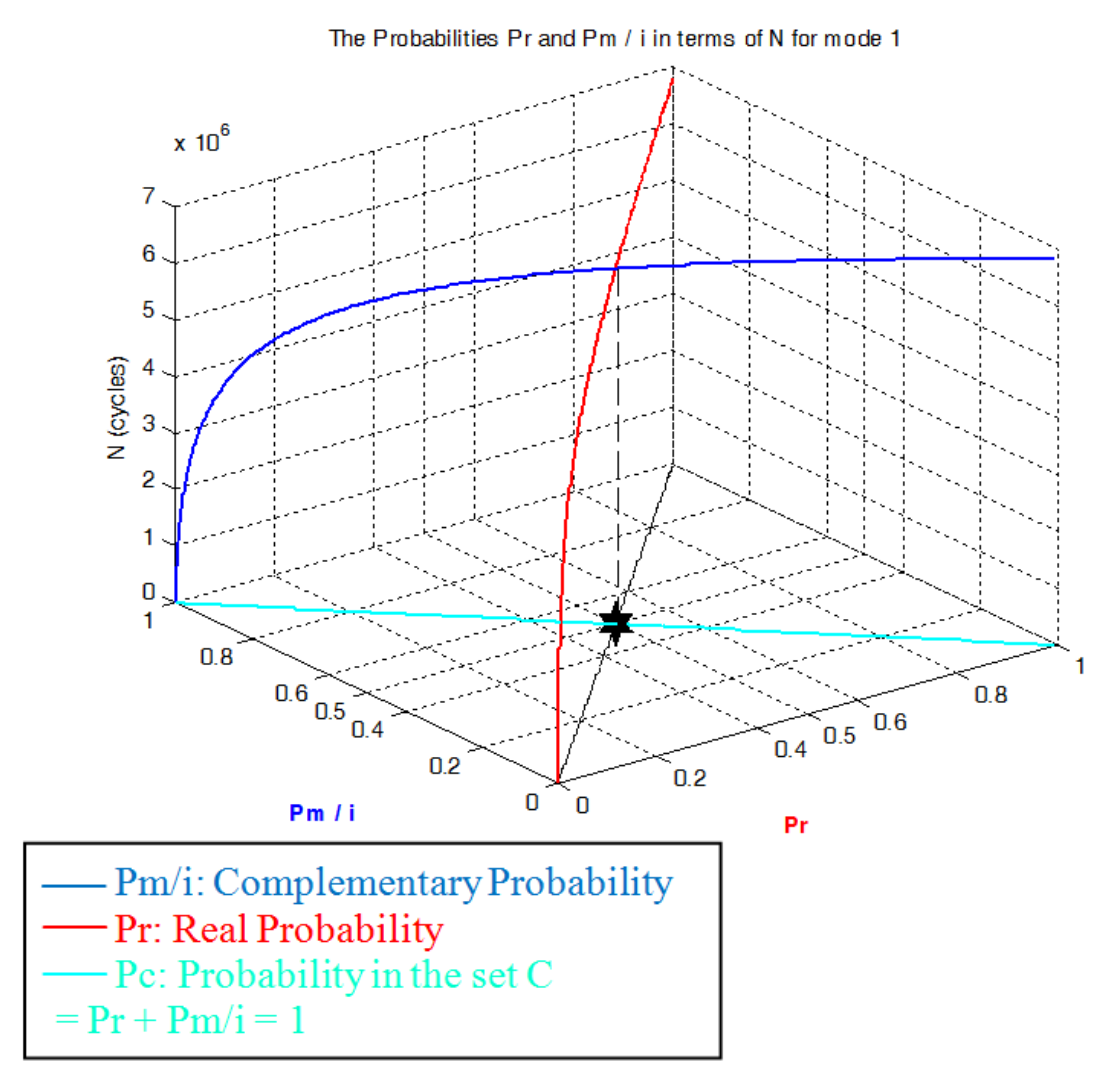

Fig. 22. $P_{r}$ and $P_{m} / i$ in terms of $N$ for mode 1

Afterward, $0<N<N_{C}, R U L(N)=N_{C}-N$ with $P_{r}(N)=$ $\psi_{2} \times[D(N)-D(N-1)] \neq 0$ and $P c(N)=P_{r}(N)+P_{m} / i(N)=1$ and $M C h f$ starts to increase during the functioning due to the environment and intrinsic conditions thus leading to a decrease in $D O K$.

If $N=N_{C} / 2=10,850,000 / 2=5,425,000$ cycles $=$ half life of the suspension system, then $R U L=N_{C}-N / 2=$ $10,850,000-5,425,000$ cycles $=5,425,000=N_{C} / 2, D=$ $0.0846, D O K=0.9342, C h f=-0.0658, M C h f=0.0658$, $P_{r}=0.0341, P_{m} / i=0.9659$, with $P_{C}=P_{r}+P_{m} / i=$ $0.0341+0.9659=1$.

If $D=0.5$ then $N=9,573,100$ cycles, $R U L=N_{C}-N=$ $1,276,900$ cycles $=N_{C} / 8.5, D O K=0.5403, C h f=-0.4597$, $M C h f=0.4597, P_{r}=0.3581, P_{m} / i=0.6419$, with $P c=$ $P_{r}+P_{m} / i=0.3581+0.6419=1$. At this point, both the rescaled $R U L$ and $D$ intersect. We can see that with the increase of $N$ and hence the decrease of $R U L$, the probability of failure $P_{\mathrm{r}}$ increases also. Furthermore, notice in the last Fig. 26 the complete symmetry at the vertical axis $D=1 / 2=$ degradation half way to complete damage.

If $N=10,004,200$ cycles (point K) both $D O K$ (minimum) and $M C h f$ (maximum) reach 0.5 where $\operatorname{RUL}(N)=N_{C}-N=10,850,000-10,004,200=845,800$ cycles $=N_{C}$ 12.83, $D=0.6355, P_{r}=0.5, P_{m} / i=0.5$ and $C h f=-0.5$, with $P c=P_{r}+P_{m} / i=0.5+0.5=1$ as always. Thus, all the EKA parameters will intersect at the point $\mathrm{K}$. We have here maximum chaos and the minimum of the system certain knowledge; therefore, the probability of the system crash is $P_{r}=$ $1 / 2=$ probability half way to complete damage. We note that relatively to mode 1 (severe road conditions), the point $\mathrm{K}$ in Fig. 23 and 24 is no more at $(6,287,900 ; 0.5)$ but shifted to the right and is now at $(10,004,200 ; 0.5)$ since the probability distribution for mode 2 (fair road conditions) is naturally different. Hence, DOK, Chf and MChf are more skewed to the left relatively to mode 1 .

If $D=0.9$ then $N=10,667,000$ cycles, $R U L=N_{C}-N=$ 183,000 cycles $=N_{C} / 59.3, D O K=0.7325, C h f=-0.2675$, MChf $=0.2675, P_{r}=0.8410, P_{m} / i=0.1590, P_{c}=P_{r}+$ $P_{m} / i=0.8410+0.1590=1$. Here, since $D=0.9$ which is very close to 1 , then the failure probability $P_{r}$ is very near to 1 since we will reach total damage very soon.

With the increase of the time of functioning, MChf and $C h f$ return to zero, $D O K$ returns to 1 where we reach total damage $(D=1)$ at $N=N_{C}=10,850,000$ cycles and hence the breakdown of the system (point L). At this last point, failure here is certain, this is the system wear out state; therefore, $P_{r}=1, P_{m} / i=0, R U L(N)=N_{C}-N=N_{C}-N_{C}$ $=0$ with $P c=P_{r}+P_{m} / i=1+0=1$ and so the logical explanation of the value of $D O K=1$ follows. 
Abdo Abou Jaoude / American Journal of Engineering and Applied Sciences 2015, 8 (1): 147.175

EKA Parameters as Functions of the Probability of Degradation for Mode 2

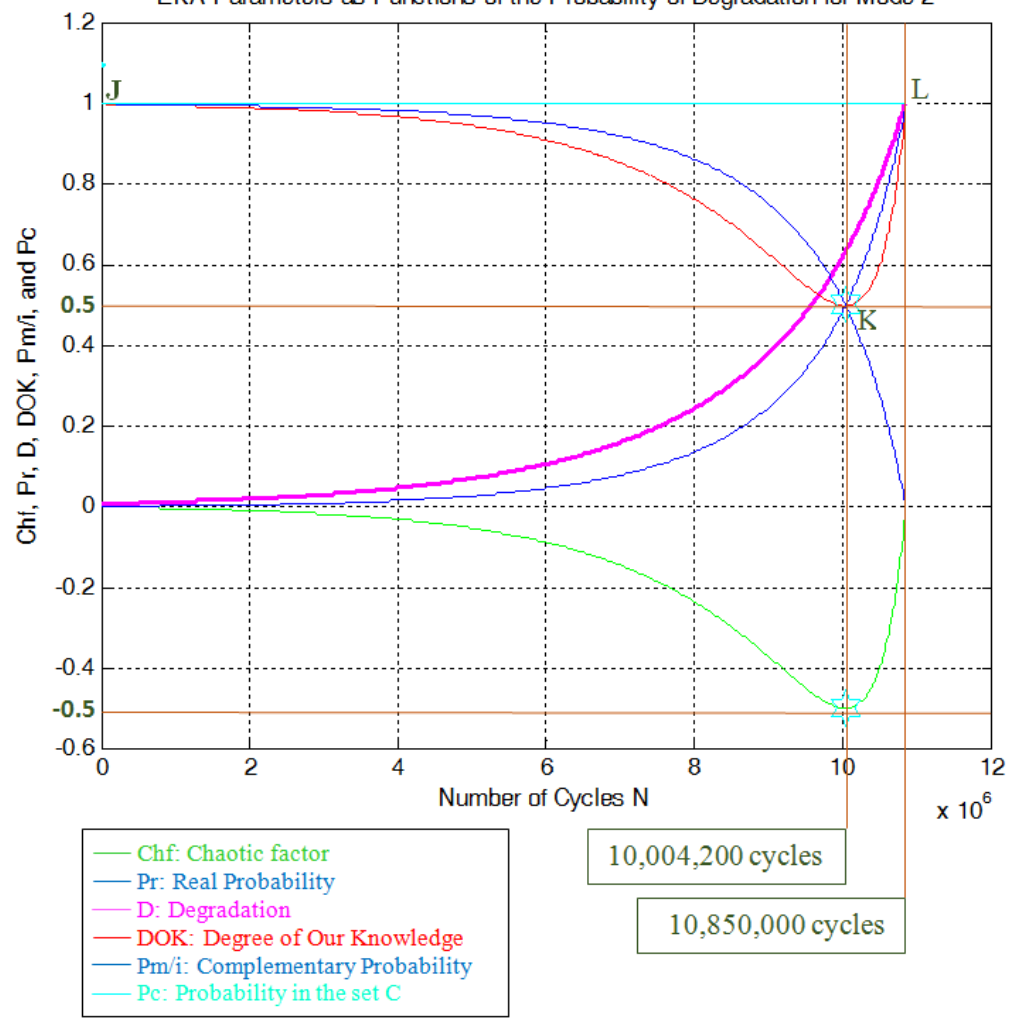

Fig. 23. Degradation and $E K A$ parameters for mode 2

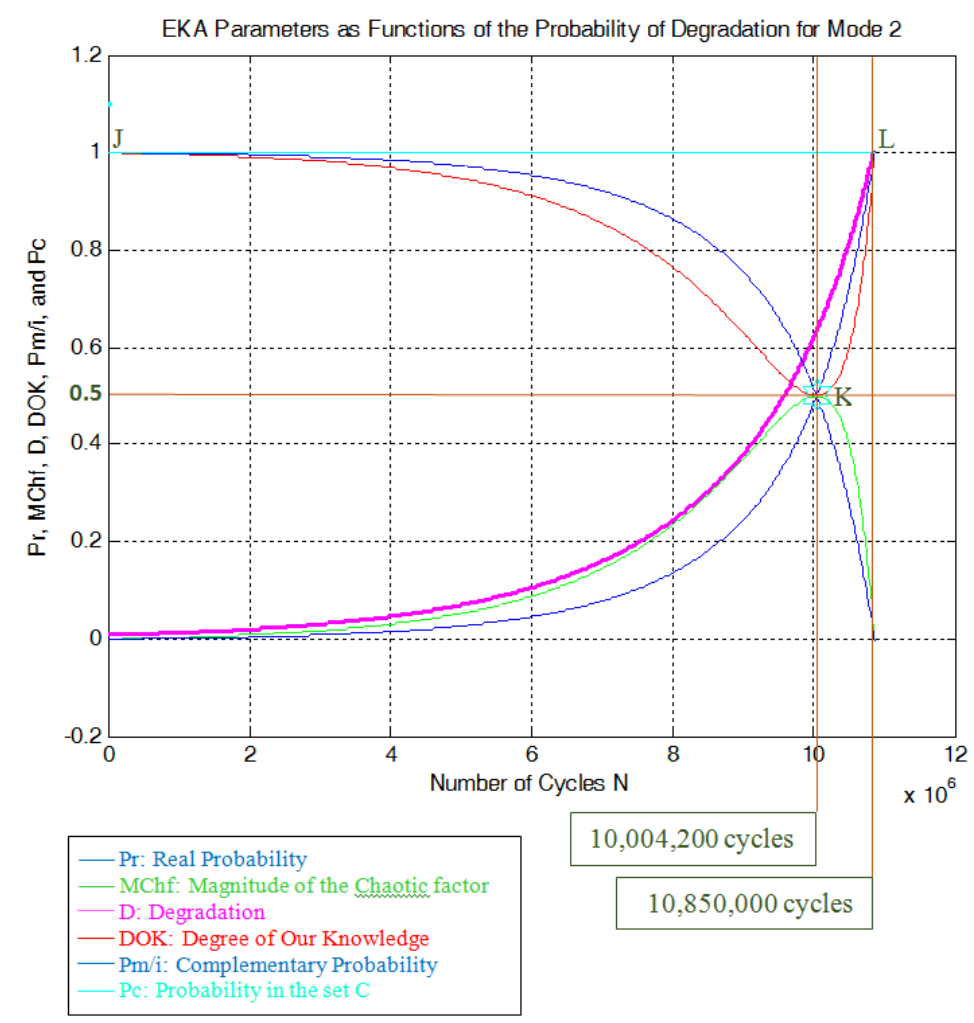

Fig. 24. Degradation and EKA parameters with $M C h f$ for mode 2 

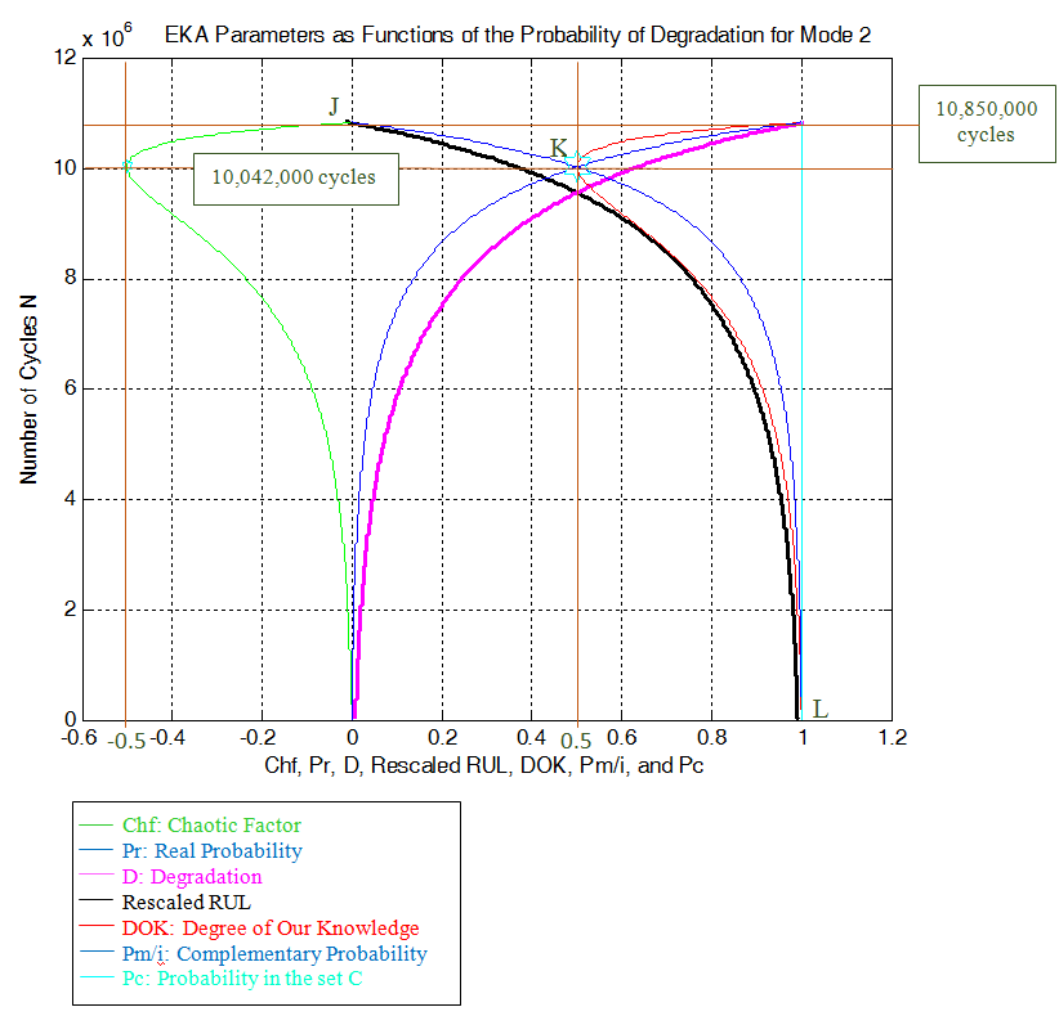

Fig. 25. Degradation, rescaled $R U L$ and $E K A$ parameters for mode 2

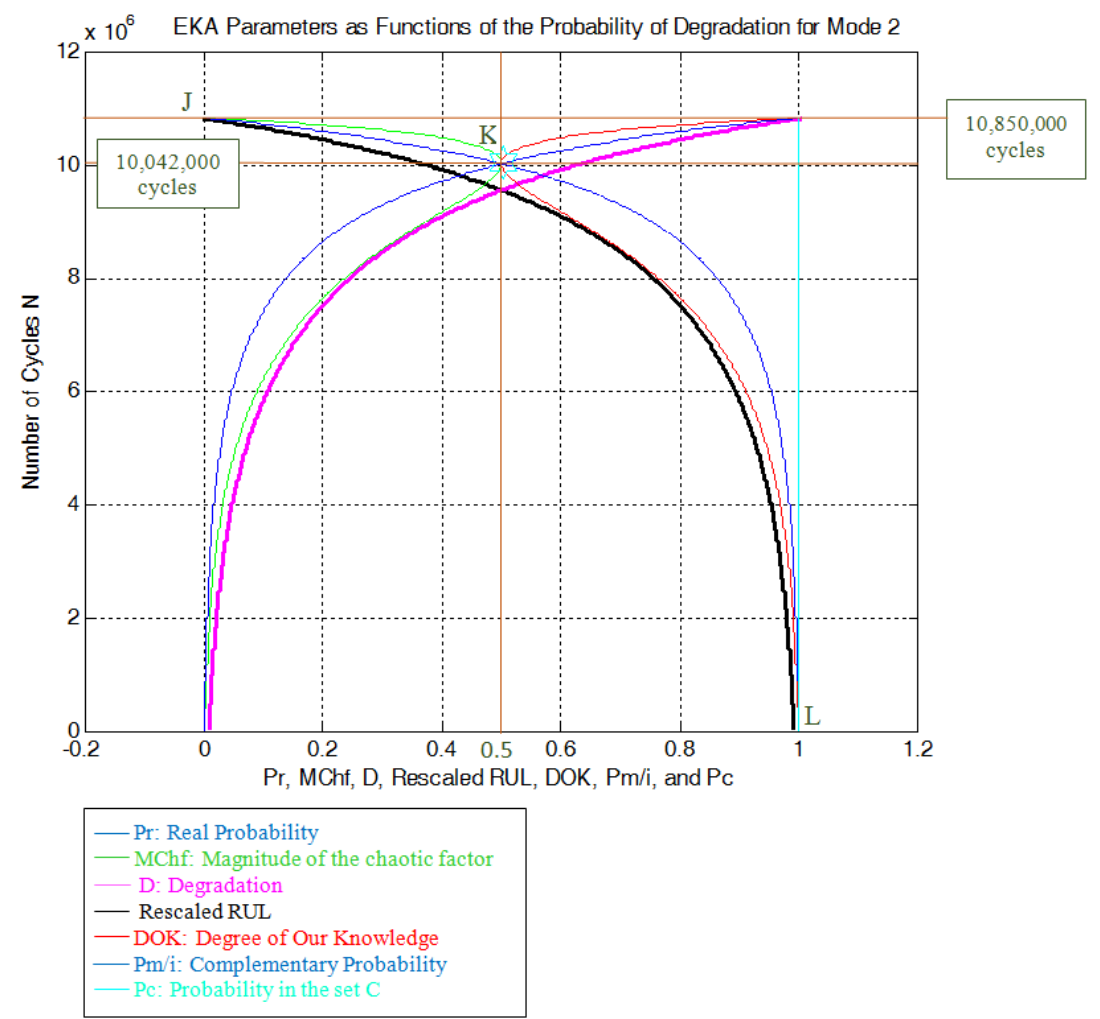

Fig. 26. Degradation, rescaled $R U L$ and $E K A$ parameters with $M C h f$ for mode 2 
EKA Parameters as Functions of the Probability of Degradation for Mode 3

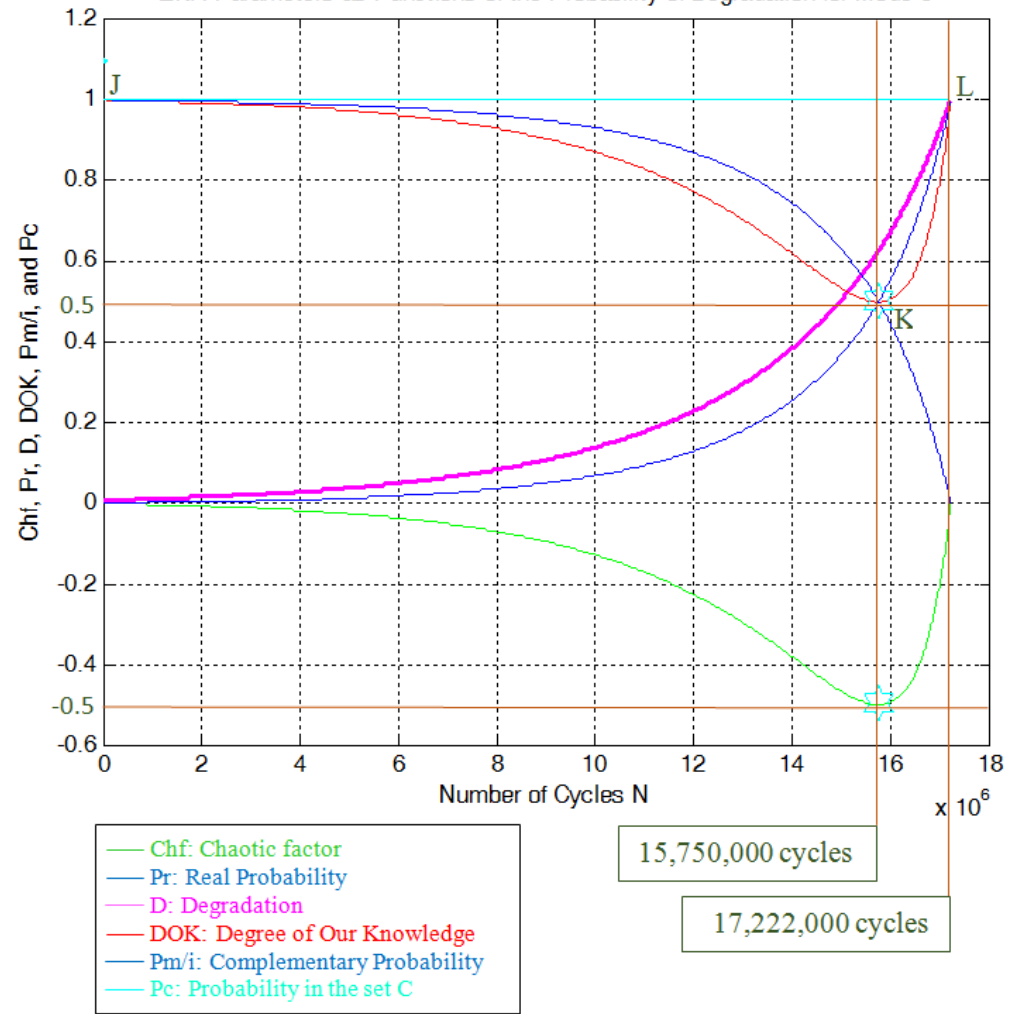

Fig. 27. Degradation and $E K A$ parameters for mode 3

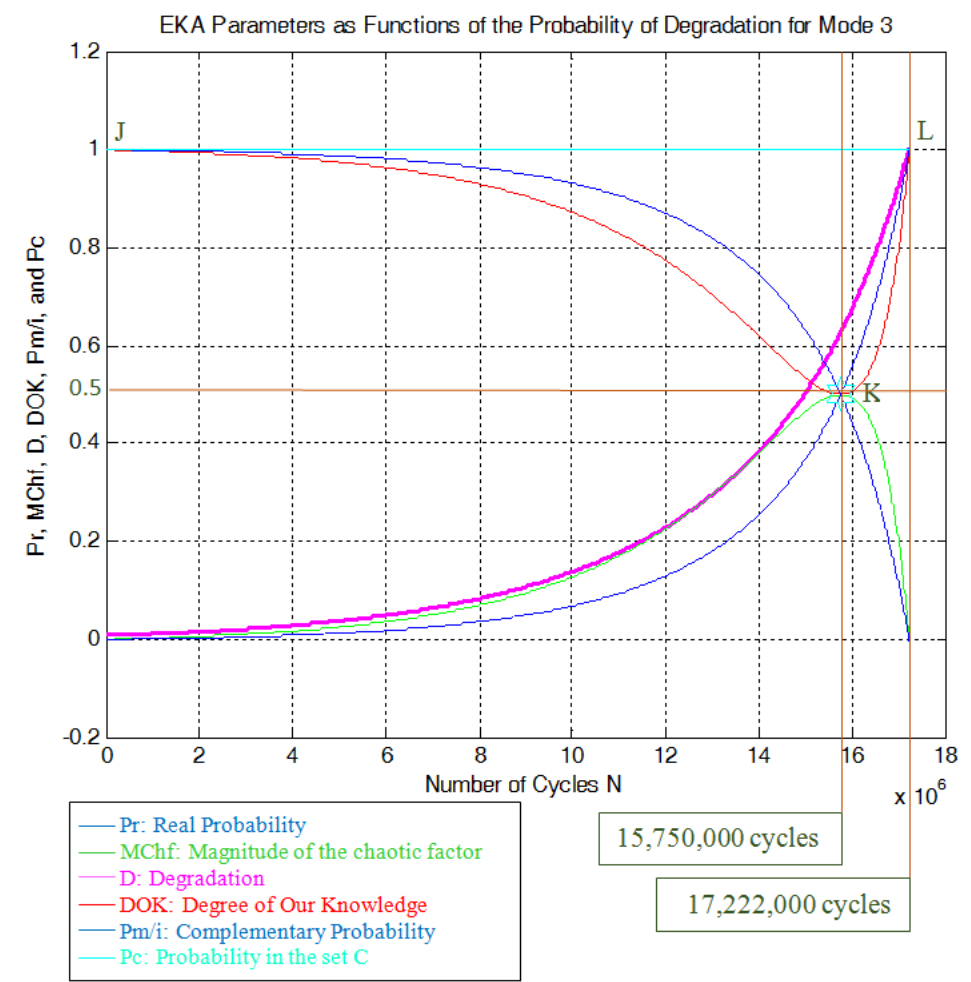

Fig. 28. Degradation and $E K A$ parameters with $M C h f$ for mode 3 
Moreover, at each instant $N$, the remaining useful lifetime $R U L(N)$ is certainly predicted in the complex set $\mathrm{C}$ with $P c=D O K-C h f=D O K+M C h f$ maintained as equal to one through a continuous compensation between $D O K$ and $C h f$. This compensation is from instant $N=0$ where $D(N)=0$ until the failure instant $N_{C}$ where $D\left(N_{C}\right)=1$. We can understand now that $D O K$ is the measure of our certain knowledge (100\% probability) about the expected event, it does not include any uncertain knowledge (with a probability less than $100 \%$ ).

We note that the same logic and analysis for mode 1 of road are applied to mode 2 of road concerning the degradation, the remaining useful lifetime, as well as all the $E K A$ parameters.

\section{The Parameters Analysis in the Suspension Prognostic for Mode 3}

Just like for modes 1 and 2 simulations, we rescaled $R U L=[0 ; 17,222,000]$ to $[0 ; 1]$ in order to fit and represent it with all the $E K A$ parameters and $D$ which vary in $[0 ; 1]$ on the same graph while having all of them as functions of the number of cycles $N=[0 ; 17,222,000]$.

We note from the Fig $27-30$ that the $D O K$ is maximum $(D O K=1)$ when $M C h f$ is minimum $(M C h f=$ 0 ) (points $J$ and $L$ ) and that means when the magnitude of the chaotic factor (MChf) decreases our certain knowledge increases.

At the beginning (point $\mathrm{J}) P_{r}(N=0)=0$, the system is intact (zero damage: $D=0$ ) and has zero chaotic factor $(C h f(0)=M C h f(0)=0)$ before any usage, at this instant (cycles number) $\operatorname{DOK}(0)=1$ and $R U L(0)=N_{C}-0=N_{C}=$ $17,222,000$ cycles $=N_{C} / 1$. Here $P_{m} / i(0)=1$ with $P c(0)=$ $P_{r}(0)+P_{m} / i(0)=0+1=1$.

Afterward, $0<N<N_{C}, R U L(N)=N_{C}-N$ with $P_{r}(N)=$ $\psi_{3} \times[D(N)-D(N-1)] \neq 0$ and $P c(N)=P_{r}(N)+P_{m} / i(N)=1$ and $M C h f$ starts to increase during the functioning due to the environment and intrinsic conditions thus leading to a decrease in $D O K$.

If $N=N_{C} / 2=17,222,000 / 2=8,611,000$ cycles $=$ half life of the suspension system, then $R U L=N_{C}-N / 2=$ $17,222,000-8,611,000$ cycles $=8,611,000=N_{C} / 2, D=$ $0.0977, D O K=0.9157, C h f=-0.0843, M C h f=0.0843$, $P_{r}=0.0441, P_{m} / i=0.9559$, with $P_{c}=P_{r}+P_{m} / i=$ $0.0441+0.9559=1$.

If $D=0.5$ then $N=14,947,000$ cycles, $R U L=N_{\mathrm{C}}-N=$ 2,275,000 cycles $=N_{\mathrm{C}}$ 7.6, DOK $=0.5383, \mathrm{Chf}=-0.4617$, $M C h f=0.4617, P_{r}=0.3616, P_{m} / i=0.6384$, with $P \mathrm{c}=P_{\mathrm{r}}+$ $P_{m} / i=0.3616+0.6384=1$. At this point, both the rescaled $R U L$ and $D$ intersect. We can see that with the increase of $N$ and consequently the decrease of $R U L$, the probability of failure $P_{r}$ increases also. Furthermore, notice in the last figure (Fig. 28) the complete symmetry at the vertical axis $D=1 / 2=$ degradation half way to complete damage.

If $N=15,750,000$ cycles (point $\mathrm{K}$ ) both $D O K$ (minimum) and MChf (maximum) reach 0.5 where $R U L(N)=N_{C}-N=17,222,000-15,750,000=1,472,000$ cycles $=N_{C}$ 111.7, $D=0.6303, P_{r}=0.5, P_{m} / i=0.5$ and $C h f=-0.5$ with $P c=P_{r}+P_{m} / i=0.5+0.5=1$ as always. Hence, all the $E K A$ parameters will intersect at the point $\mathrm{K}$. We have here maximum chaos and the minimum of the system knowledge; therefore, the probability of the system crash is $P_{r}=1 / 2=$ probability half way to complete damage. We note that relatively to mode 1 (severe road conditions) and mode 2 (fair road conditions), the point $\mathrm{K}$ in Fig. 27 and 28 is no more at $(6,287,900 ; 0.5)$ or $(10,004,200 ; 0.5)$ but shifted more to the right and is now at $(15,750,000 ; 0.5)$ since the probability distribution for mode 3 (good road conditions) is eventually different. Hence, $D O K, C h f$ and $M C h f$ are more negatively skewed relatively to modes 1 and 2 .

If $D=0.9$ then $N=16,885,000$ cycles, $R U L=N_{C}-N=$ 337,000 cycles $=N_{C} / 51.1, D O K=0.7308, C h f=-0.2692$, $M C h f=0.2692, P_{r}=0.8397, P_{m} / i=0.1603$, with $P_{c}=P_{r}$ $+P_{m} / i=0.8397+0.1603=1$. Here, since $D=0.9$ which is very close to 1 , then the failure probability $P_{\mathrm{r}}$ is very near to 1 since we will reach total damage very soon.

With the increase of the time of functioning, MChf and $C h f$ return to zero, $D O K$ returns to 1 where we reach total damage $(D=1)$ at $N=N_{C}=17,222,000$ cycles and hence the breakdown of the system (point L). At this last point, failure here is certain, this is the system wear out state; therefore, $P_{r}=1, P_{m} / i=0, \operatorname{RUL}(N)=N_{C^{-}} N=N_{C^{-}}$ $N_{C}=0$ with $P c=P_{r}+P_{m} / i=1+0=1$ and so the logical explanation of the value of $D O K=1$ follows.

Moreover, at each instant $N$, the remaining useful lifetime $\operatorname{RUL}(N)$ is certainly predicted in the complex set $\mathrm{C}$ with $P \mathrm{c}$ maintained as equal to one through a continuous compensation between $D O K$ and Chf. This compensation is from instant $N=0$ where $D(N)=0$ until the failure instant $N_{C}$ where $D\left(N_{C}\right)=1$, keeping always $P c=D O K-C h f=D O K+M C h f=1$. Furthermore, what is truly interesting in three modes simulations is that we have quantified both the degree of our knowledge and the chaotic factor of the vehicle suspension system.

It is clear from the three modes simulations that $D O K$ is the measure of our certain knowledge (100\% probability) about the expected event and it does not include any uncertain knowledge (with a probability less than 100\%).

We note that the same methodology and analysis for mode 1 and 2 of roads are applied to mode 3 of road concerning the degradation, the remaining useful lifetime, as well as all the $E K A$ parameters. Thus, we can consequently conclude that whatever the road conditions are, both the logic and the method implemented are similar. This proves the validity of the new axioms developed and the novel prognostic model adopted.

\section{The Parameters Visualization in the Suspension Prognostic for the Three Modes}

Furthermore, the following simulations (Fig. 31-34) recapitulate all the previous figures. 

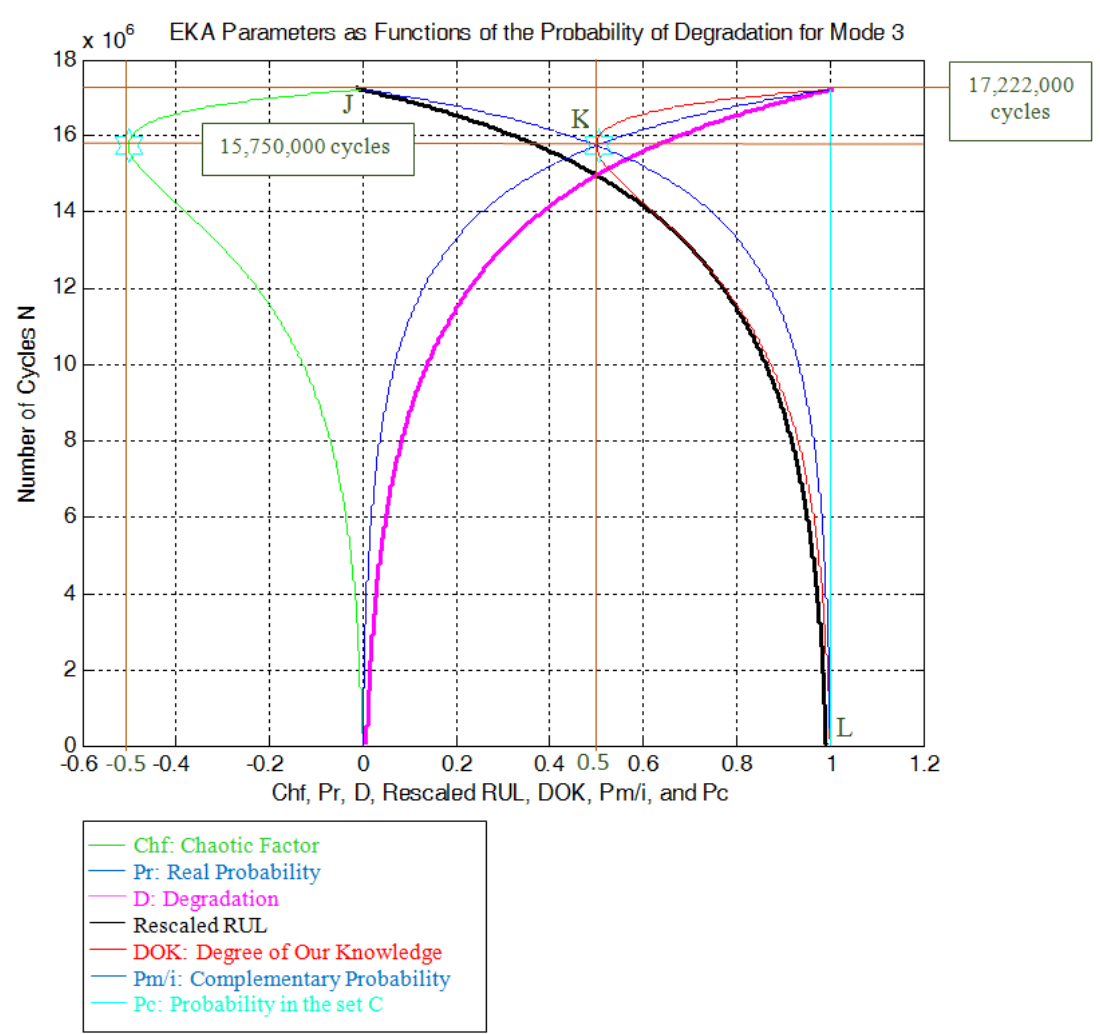

Fig. 29. Degradation, rescaled $R U L$ and $E K A$ parameters for mode 3

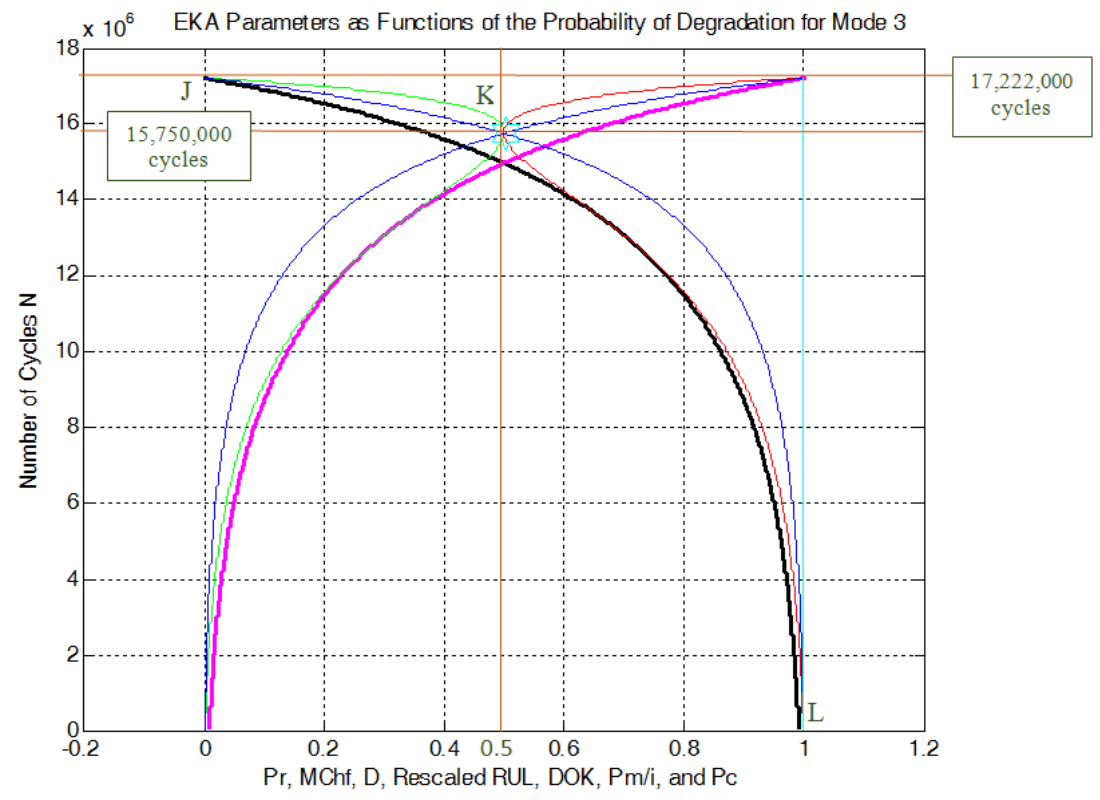

— Pr: Real Probability
- MChf: Magnitude of the chaotic factor
— D: Degradation
— Rescaled RUL
— DOK: Degree of Our Knowledge
— Pm/i: Complementary Probability
- Pc: Probability in the set C

Fig. 30. Degradation, rescaled $R U L$ and $E K A$ parameters with $M C h f$ for mode 3 
EKA Parameters as Functions of the Probability of Degradation for the Three Modes

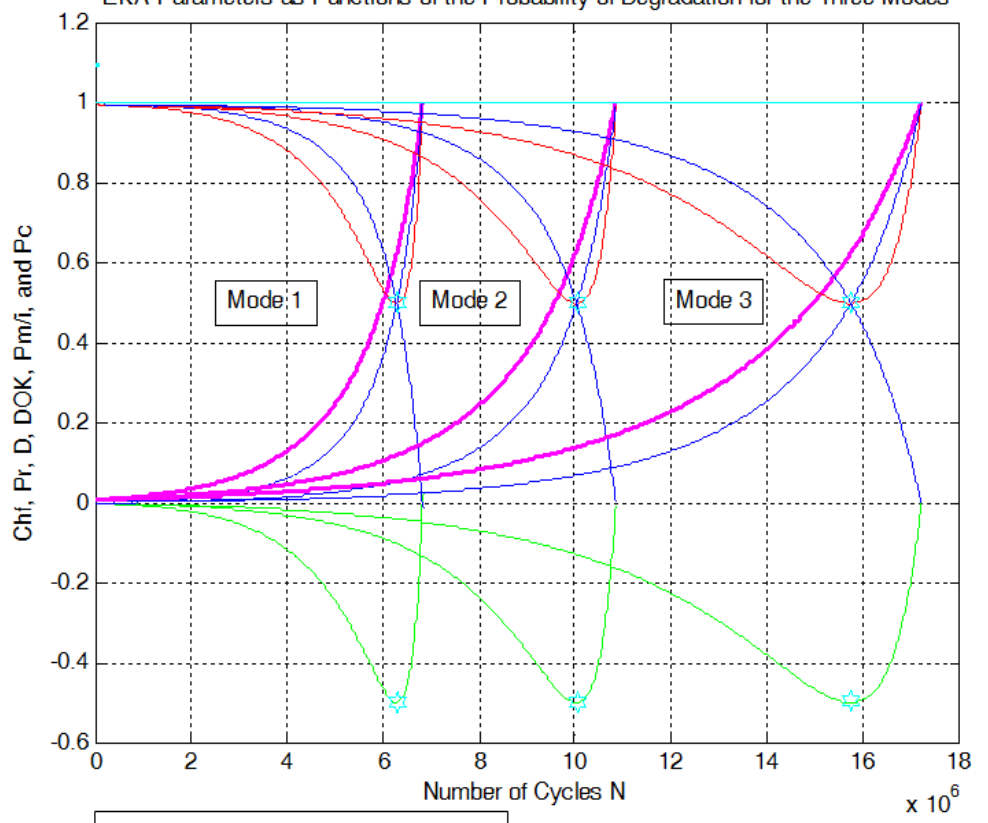

$$
\begin{aligned}
& \text { - Chf: Chaotic factor } \\
& \text { - Pr: Real Probability } \\
& \text { D: Degradation } \\
& \text { - DOK: Degree of Our Knowledge } \\
& \text { - Pm/i: Complementary Probability } \\
& \text { — Pc: Probability in the set C }
\end{aligned}
$$

Fig. 31. Degradation and EKA parameters for the three modes

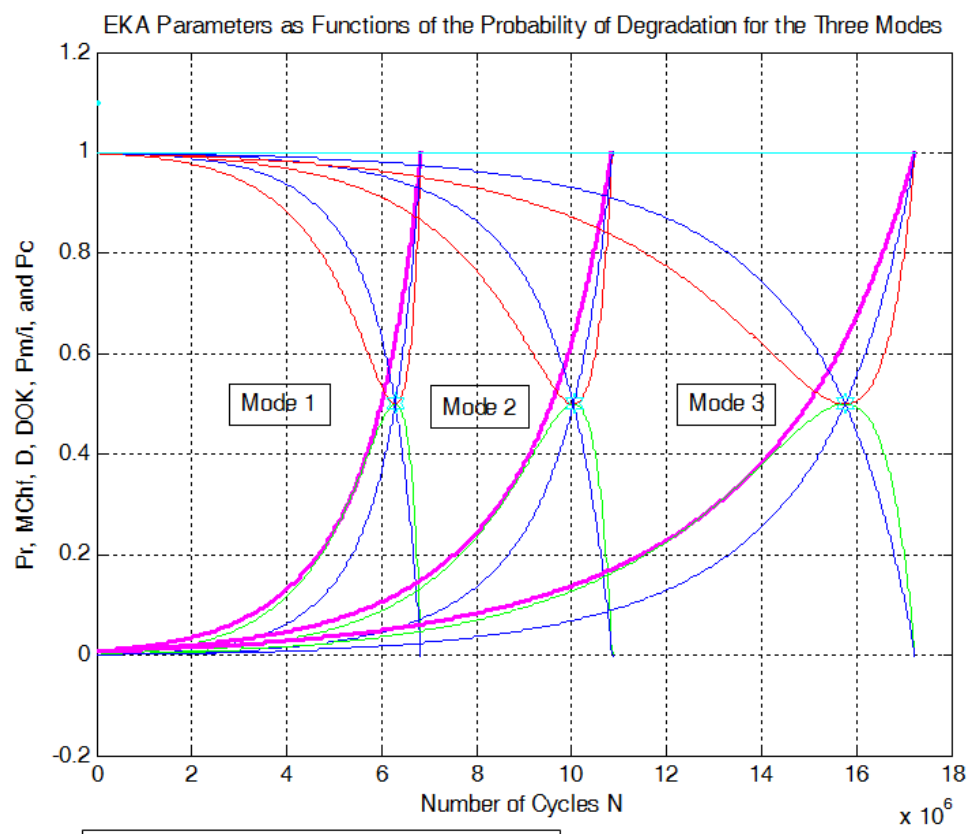

$$
\begin{aligned}
& \text { — Pr: Real Probability } \\
& \text { - MChf: Magnitude of the chaotic factor } \\
& \text { - D: Degradation } \\
& \text { — DOK: Degree of Our Knowledge } \\
& \text { - Pm/i: Complementary Probability } \\
& \text { - Pc: Probability in the set C }
\end{aligned}
$$

Fig. 32. Degradation and $E K A$ parameters with $M C h f$ for the three modes 


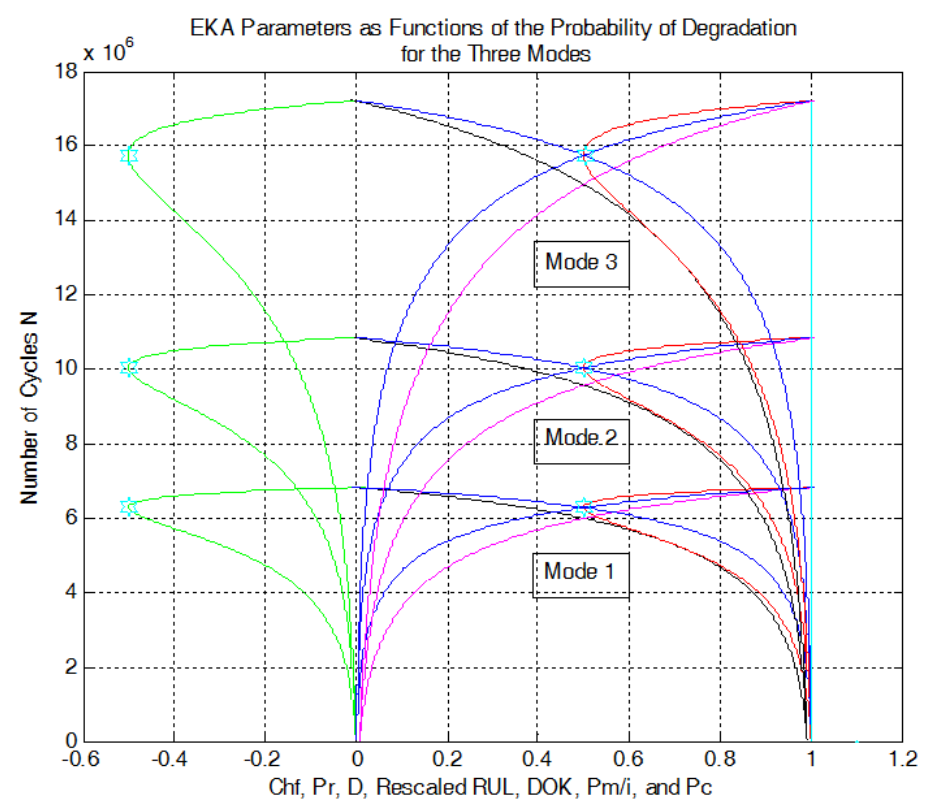

- Chf: Chaotic Factor
- Pr: Real Probability
- D: Degradation
- Rescaled RUL
— DOK: Degree of Our Knowledge
- Pm/i: Complementary Probability
- Pc: Probability in the set C

Fig. 33. Degradation, rescaled $R U L$ and $E K A$ parameters for the three modes

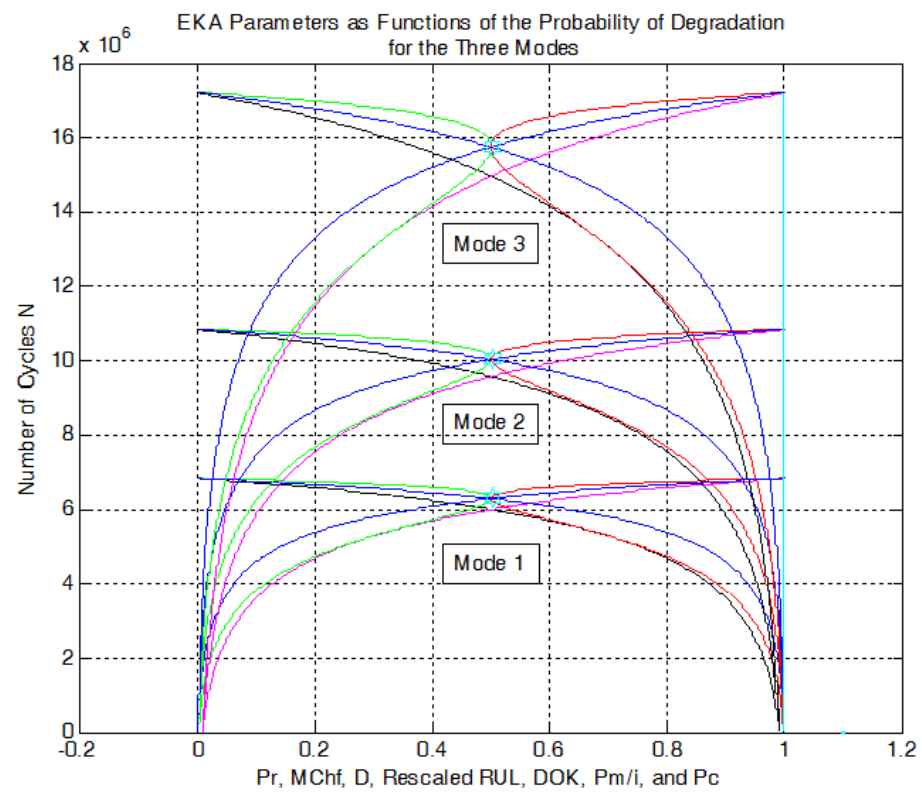

$$
\begin{aligned}
& \text { - Pr: Real Probability } \\
& \text { - MChf: Magnitude of the chaotic factor } \\
& \text { - D: Degradation } \\
& \text { — Rescaled RUL } \\
& \text { - DOK: Degree of Our Knowledge } \\
& \text { - Pm/i: Complementary Probability } \\
& \text { Pc: Probability in the set C }
\end{aligned}
$$

Fig. 34. Degradation, rescaled $R U L$ and $E K A$ parameters with $M C h f$ for the three modes 


\section{Conclusion and Perspectives}

The analytic linear prognostic model elaborated in a previous work permits to predict, at each cycle or instant, the remaining useful lifetime of the system by a simple and practical way. The lifetimes are concluded from the time reading at each instant on the degradation curves or trajectories. To show the efficiency of this prognostic model, it was applied in simulation to predict the fatigue life of the vehicle suspension systems. The degradation trajectories deduced allowed us to determine their remaining useful lifetimes. The proposed model belongs to the modelbased approach. Whenever the analytic damage laws are available, like the linear damage propagation law of Palmgren-Miner used in my previous and current papers, this model can be adaptable to new situations or cases. In industrial systems, this model shows that it is convenient and practical as a flexible tool for prognostic analysis.

In the current paper I applied the theory of Extended Kolmogorov Axioms to analytic and linear prognostic of vehicle suspension system. Hence, I established a tight link between the new paradigm and degradation or the remaining useful lifetime. Thus, I developed the theory of "Complex Probability" beyond the scope of my previous four papers on this topic. Although the analytic laws used previously are deterministic and very well-known but there are random factors (temperature, humidity, applied load location, water action, etc...) that affect the system and make its degradation function deviate from its calculated trajectory predefined by these laws. An updated follow-up of the degradation behavior with time or cycle number is done by the failure probability $P_{r}$ due to its definition that evaluates the jumps in $D$. In fact, an accentuated effect of chaos on the system leads to a bigger jump in the degradation trajectory and hence to a greater probability of failure. As it was proved and illustrated, when the degradation index is 0 or 1 and correspondingly the $R U L$ is $N_{C}$ or 0 then the Degree of Our Knowledge $(D O K)$ is one and the chaotic factor (Chf and $M C h f)$ is 0 since the state of the system is totally known. During the process of degradation $(0<D<1)$ we have: $0.5<D O K<1,-0.5<$ $C h f<0$ and $0<M C h f<0.5$. Notice that during the whole process of degradation we have $P \mathrm{c}=D O K-C h f$ $=D O K+M C h f=1$, that means that the phenomenon which seems to be random and stochastic in $\mathrm{R}$ is now deterministic and certain in $\mathrm{C}=\mathrm{R}+\mathrm{M}$ and this after adding to $\mathrm{R}$ the contributions of $\mathrm{M}$ and hence after subtracting the chaotic factor from the degree of our knowledge. Moreover, for each value of an instant $N$, I have determined its corresponding probability of failure which is a function of the degradation jump. Therefore, at each cycle $N, R U L(N)=N_{C}-N$ is certainly predicted in the complex set $C$ with $P c$ maintained as equal to one through a continuous compensation between $D O K$ and $C h f$. This compensation is from instant $N=0$ where $D(N)=0$ until the failure instant $N_{\text {C }}$ where $D\left(N_{C}\right)=1$. Furthermore, using all these graphs illustrated throughout the whole paper, we can visualize and quantify both the system chaos (Chf and $M C h f)$ and the system certain knowledge ( $D O K$ and $P c$ ). This is certainly very interesting and fruitful and shows once again the benefits of extending Kolmogorov's axioms and thus the originality and usefulness of this new field in applied mathematics and prognostic that can be called verily: "The Complex Probability Paradigm".

As prospective and future works, it is planned to more develop the novel proposed prognostic methodology and to apply it to a wide set of dynamic systems. In addition, the nonlinear damage propagation law will be considered as well as the extended stochastic model which is based on the accumulation of damage due to fatigue crack propagation in stochastic conditions where the initial crack length and the loading are taken as random.

\section{Ethics}

This article is original and contains unpublished material. The corresponding author confirms that all of the other authors have read and approved the manuscript and no ethical issues involved.

\section{References}

Abou Jaoude, A., K. El-Tawil, S. Kadry, H. Noura and M. Ouladsine, 2010a. Analytic prognostic model for a dynamic system. Int. Rev. Automatic Control, 3: 568-577.

Abou Jaoude, A., K. El-Tawil and S. Kadry, 2010b. Prediction in complex dimension using Kolmogorov's set of axioms. J. Math. Stat., 6: 116-124. DOI: 10.3844/jmssp.2010.116.124

Abou Jaoude, A., S. Kadry, K. El-Tawil, H. Noura and M. Ouladsine, 2011. Analytic prognostic for petrochemical pipelines. J. Mech. Eng. Res., 3: 64-74.

Abou Jaoude, A., 2012. Advanced analytical model for the prognostic of industrial systems subject to fatigue. Ph.D. Thesis, Aix-Marseille Université.

Abou Jaoude, A. and K. El-Tawil, 2013a. Analytic and nonlinear prognostic for vehicle suspension systems. Am. J. Eng. Applied Sci., 6: 42-56. DOI: 10.3844/ajeassp.2013.42.56

Abou Jaoude, A. and K. El-Tawil, 2013b. Stochastic prognostic paradigm for petrochemical pipelines subject to fatigue. Am. J. Eng. Applied Sci., 6: 145-160. DOI: 10.3844/ajeassp.2013.145.160 
Abou Jaoude, A., 2013a. Automatic Control and Prognostic. 1st Edn., Scholars' Press, ISBN-13: 9783639703351, pp: 240.

Abou Jaoude, A., 2013b. The complex statistics paradigm and the law of large numbers. J. Math. Stat., 9: 289-304. DOI: 10.3844/jmssp.2013.289.304

Abou Jaoude, A., 2013c. The theory of complex probability and the first order reliability method. J. Math. Stat., 9: 310-324. DOI: 10.3844 jmssp.2013.329.343

Abou Jaoude, A., 2014. Complex probability theory and prognostic. J. Math. Stat., 10: 1-24. DOI: $10.3844 /$ jmssp.2014.1.24

Abou Jaoude, A., 2015. Analytic and linear prognostic model for a vehicle suspension system subject to fatigue. Syst. Sci. Control Eng., 3: 81-98. DOI: $10.1080 / 21642583.2014 .987359$

Bell, E.T., 1992. The Development of Mathematics. 1st Edn., Dover Publications, Inc., USA, ISBN10: 0486272397, pp: 617.

Benton, W., 1966a. Probability, encyclopedia Britannica. Encyclopedia Britannica Inc., Chicago.

Benton, W., 1966b. Mathematical probability, encyclopedia Britannica. Encyclopedia Britannica Inc., Chicago.

Boursin, J.L., 1986. Les Structures du Hasard. 1st Edn., Editions du Seuil. ISBN-10: 2020092352, pp: 186.

Dacunha-Castelle, D., 1996. Chemins de L'aleatoire: Le Hasard et le Risque Dans la Societe Moderne. 1st Edn., Flammarion, ISBN-10: 2082112268, pp: 256.

Dalmedico-Dahan, A. and J. Peiffer, 1986. Une histoire des mathématiques. Edition du Seuil.

Dalmedico-Dahan, A., J.L. Chabert and K. Chemla, 1992. Chaos Et Déterminisme. Edition du Seuil.

Ekeland, I., 1991. Au hasard: La Chance, la Science, et le Monde. 1st Edn., Editions du Seuil, ISBN-10: 2020128772, pp: 198.

El-Tawil, K. and A. Abou Jaoude, 2013. Stochastic and nonlinear-based prognostic model. Syst. Sci. Control Eng., 1: 66-81. DOI: $10.1080 / 21642583.2013 .850754$

Feller, W., 1968. An Introduction to Probability Theory and its Applications. 3rd Edn., Wiley, New York, ISBN-10: 0471257087, pp: 509.

Gleick, J., 1997. Chaos: Making a New Science. 1st Edn., Vintage, ISBN-10: 0749386061, pp: 380.

Gullberg, J., 1997. Mathematics: From the Birth of Numbers. 1st Edn., W.W. Norton, New York, ISBN-10: 039304002X, pp: 1093.

Lemaitre, J. and J. Chaboche, 1990. Mechanics of Solid Materials. 1st Edn., Cambridge University Press, New York, ISBN-10: 1107392985.

Montgomery, D.C. and G.C. Runger, 2003. Applied Statistics and Probability for Engineers. 3rd Edn., John Wiley and Sons, Inc.
Srinivasan, S.K. and K.M. Mehata, 1988. Stochastic Processes. 2nd Edn., McGraw-Hill, New Delhi.

Stewart, I., 2002. Does God Play Dice? The New Mathematics of Chaos. 2nd Edn., Wiley-Blackwell, Oxford, ISBN-10: 0631232516, pp: 416.

SCIENCE et VIE, 1999. Le mystère des mathématiques. Numéro.

Vachtsevanos, G., F. Lewis, M. Roemer, A. Hess and B. $\mathrm{Wu}, 2006$. Intelligent Fault Diagnosis and Prognosis for Engineering Systems. 1st Edn., John Wiley and Sons, Inc., Hoboken, ISBN-10: 047172999X, pp: 456.

Van Kampen, N.G., 2006. Stochastic Processes in Physics and Chemistry. 1st Edn., North Holland, ISBN-10: 0444529659, pp: 464.

Walpole, R., R. Myers, S. Myers and K. Ye, 2002. Probability and Statistics for Engineers and Scientists. 7th Edn., Prentice Hall.

\section{Nomenclature}

$\mathrm{R} \quad=$ Real set of events

$\mathrm{M}=$ Imaginary set of events

$\mathrm{C}=$ Complex set of events

$i=$ The imaginary number where $i^{2}=-1$

$E K A=$ Extended Kolmogorov's Axioms

$P_{\text {rob }}=$ probability of any event

$P_{\mathrm{r}} \quad=$ Probability in the real set $R$

$P_{\mathrm{m}}=$ Probability in the imaginary set $M$ corresponding to the real probability in $R$

$P \mathrm{c}=$ Probability of an event in $R$ with its associated event in $M$

$=$ Probability in the complex set $C$

$Z=$ Complex probability number and vector $=$ sum of $P_{r}$ and $P_{m}$

$D O K=|Z|^{2}=$ Degree of Our Knowledge of the random experiment and event

Chf = Chaotic factor

$M C h f=$ Magnitude of the Chaotic factor

$N=$ Number of load cycles

$N_{\mathrm{C}}=$ Number of cycles till system failure

$D=$ Degradation of a system

$R U L=$ Remaining Useful Lifetime of a system 